\title{
Which is the best treatment of pediatric upper urinary tract stones among extracorporeal shockwave lithotripsy, percutaneous nephrolithotomy and retrograde intrarenal surgery: a systematic review
}

Qing $\mathrm{He}^{\dagger}$, Kaiwen Xiao ${ }^{\dagger}$, Yuntian Chen, Banghua Liao, Hong Li and Kunjie Wang* (1)

\begin{abstract}
Background: Although the indications of minimally invasive treatments for pediatric urolithiasis are similar to those in adults, it is still crucial to make the right treatment decision due to the special considerations of children. This review aims to evaluate the efficacy and safety of extracorporeal shockwave lithotripsy (SWL), percutaneous nephrolithotomy (PCNL), and retrograde intrarenal surgery (RIRS) in the management of pediatric upper urinary tract stones.

Methods: EMBASE, PubMed, and the Cochrane Library were searched from their first available date to March 2018. The studies that meet the inclusive criteria were included. The efficacy and safety of the treatments were assessed by means of meta-analysis of the stone free rate (SFR), complication rate, effectiveness quotient (EQ) and secondary outcome indicators.

Results: A total of 13 comparative studies were identified for data analysis. PCNL presented a significantly higher SFR compared with SWL. Similarly, the single-session SFR of RIRS was significantly higher than SWL. However, no significant difference was found between RIRS and SWL in the overall SFR. There was no significant difference between PCNL and RIRS in the SFR. Furthermore, no significant differences in complication rates were found among the three therapies. Compared with the other two treatments, PCNL had a longer operative time, fluoroscopy time and hospital stay. SWL had a shorter hospital stay, higher retreatment rate and auxiliary rate in comparison with the other two treatments. The present data also showed that PCNL presented a higher EQ than the other two treatments, and RIRS had a lower efficiency than SWL and PCNL. In the subgroup analysis of pediatric patients with stone $\leq 20 \mathrm{~mm}$, the comparative results were similar to those described above, except for the higher complication rate of PCNL than SWL.

(Continued on next page)
\end{abstract}

\footnotetext{
* Correspondence: wangkj@scu.edu.cn

${ }^{\dagger}$ Qing He and Kaiwen Xiao contributed equally to this work.

Department of Urology, Institute of Urology (Laboratory of Reconstructive

Urology), West China Hospital, Sichuan University, No. 37 Guo Xue Xiang,

Chengdu, Sichuan 610041, People's Republic of China
}

(c) The Author(s). 2019 Open Access This article is distributed under the terms of the Creative Commons Attribution 4.0 International License (http://creativecommons.org/licenses/by/4.0/), which permits unrestricted use, distribution, and reproduction in any medium, provided you give appropriate credit to the original author(s) and the source, provide a link to the Creative Commons license, and indicate if changes were made. The Creative Commons Public Domain Dedication waiver (http://creativecommons.org/publicdomain/zero/1.0/) applies to the data made available in this article, unless otherwise stated. 
(Continued from previous page)

Conclusions: Although SWL as an outpatient procedure provides shorter hospital stay and reduces operative time, it has a lower SFR and higher retreatment rate than the other two treatments. PCNL exhibits a higher SFR and EQ than SWL; nevertheless, it has a longer operative time and fluoroscopy time than the other two procedures. RIRS offers a similar SFR as PCNL but a lower efficiency than PCNL.

Keywords: Extracorporeal shockwave lithotripsy, Pediatric, Percutaneous nephrolithotomy, Retrograde intrarenal surgery, upper urinary tract stone

\section{Background}

Pediatric urolithiasis is an important global medical issue, particularly in regards to the selection of different treatments by care providers. The primary risk factors of forming stones among children include geographical conditions, climates, and diet customs. Additionally, the morbidity is opposite to the level of economic development; the morbidity is 1 to $5 \%$ in developed nations and 5 to $15 \%$ in developing nations [1]. Nevertheless, rates of pediatric urolithiasis have increased in developed countries. Extracorporeal shockwave lithotripsy (SWL) has long been considered as the first-line therapy for pediatric urolithiasis less than 20 $\mathrm{mm}$ [2]. There is a growing trend in the management of pediatric urolithiasis with endourologic procedures due to the technological advances and miniaturization of instruments. SWL, percutaneous nephrolithotomy (PCNL), and retrograde intrarenal surgery (RIRS) have become standard therapeutic options for adult urinary stones and can be extended to pediatric stones.

SWL is still the first choice for pediatric renal stones since it is the least-invasive approach for managing pediatric urolithiasis. The indications of SWL for pediatric urolithiasis are similar to those of adults, according to the European Association of Urology (EAU) guideline, and the stone fragments are more easily passed by pediatric patients than adult patients [3]. Similarly, the American Urological Association (AUA) guideline recommends that clinicians offer SWL or RIRS as first-line therapy for pediatric patient with a total renal stone burden under $20 \mathrm{~mm}$ [4]. However, the high retreatment rates and the potential biological effects on the immature kidneys and adjacent tissues may limit the range of application of SWL.

Age is not a limiting factor, since there are appropriately sized PCNL instruments for children, and this approach has even been reported in a 5-month-old infant $[5,6]$. In children, PCNL is recommended to manage renal stones larger than $20 \mathrm{~mm}[3,4]$, especially multiple renal stones. The most common complications are fever and bleeding with some serious cases requiring blood transfusions to prevent ischaemia. The necessary radiation exposure and its effects on pediatric renal function remain controversial [7].RIRS is considered as an ideal choice for medium and large-sized pediatric renal or ureteric stones. With their smaller diameter, excellent optical properties, relatively wider range of motion, and the multiple secure and effective lithotripsy techniques available, increasing numbers of urologists prefer to treat pediatric upper urinary tract stones using ureteroscopy $[8,9]$. Nevertheless, ureteroscopy, especially flexible ureteroscopy, is quite a challenge for clinicians who lack the necessary training. In addition, the high purchase and maintenance costs of RIRS result in this technique not being available in every medical centre.

In adults, any of the above three procedures could be recommended to patients with stones smaller than 20 $\mathrm{mm}$ located in the renal pelvis and upper or middle calices [3]. Although the indications for treatment in pediatric urolithiasis are similar to those of adults, the unique considerations, such as a smaller anatomical structure and immature kidneys, make it vital to identify the most effective and safest procedure for children. To evaluate the efficacy and safety of SWL, PCNL, and RIRS in the management of pediatric upper urinary tract stones, we performed a systematic review, synthesizing the available high-level studies.

\section{Methods}

\section{Study identification}

Literature databases including EMBASE, PubMed, and the Cochrane Library, were searched from their first available date to March 2018. The first search procedure was performed to identify all relevant trials retrieved using the following search terms: (extracorporeal shock wave lithotripsy/ESWL/SWL or percutaneous nephrolithotomy/percutaneous lithotripsy/PCNL or Retrograde intrarenal surgery/RIRS/flexible ureterorenoscopy/flexible ureteroscopy/URS/FURS) and (Pediatric/pediatrics/ child/children). The language was restricted to English. Scanning the reference lists of the selected articles to identify additional articles was also conducted.

\section{Inclusion and exclusion criteria}

The inclusion criteria were: 1) comparative studies evaluating the efficacy of SWL versus PCNL, SWL versus RIRS, PCNL versus RIRS, or SWL versus PCNL versus RIRS in the treatment of pediatric renal or upper 
ureteral calculi; and 2) the stone-free status was evaluated postoperatively by KUB film and/or ultrasonography. In addition, for the exclusion criteria were: 1) abstracts, comments, reviews, conference papers, or systematic reviews; and 2) inclusion of children with distal ureteral stones, uncontrolled coagulation disorders, active urinary tract infections, obstructive urinary tract anomalies, severe hydronephrosis or kidney failure (GFR $<15 \mathrm{~mL} / \mathrm{min}$ ). Duplicated studies were included in our research, however, only the latest data were assessed in our review.

\section{Data extraction and outcome measurement}

Two investigators reviewed the titles and abstracts identified by the search strategies. The following data were extracted from each study if available by using a Microsoft Excel worksheet: first author's name, year of publication, mean age of the patients, type of stones, stone sizes, stone free rate (SFR), complications, operative time, length of hospital stay, need for auxiliary procedures, and retreatments. We graded the complications according to the Clavien-Dindo classification of surgical complications [10]. To increase the comparability among these treatment modalities, the effectiveness quotients (EQs) were calculated according to the equation reported by Clayman and colleagues [11]. Dichotomous data were classified into two-by-two tables. For continuous data, available summary estimates per group (mean, changes in means) and measures of variability (standard deviation [SD], 95\% confidence interval $[\mathrm{CI}]$ ) were extracted.

\section{Evaluation of study quality}

The levels of evidence (LE) of all included studies were assessed by the Oxford Centre for Evidence Based Medicine-Levels of Evidence [12]. The methodological quality of the studies was evaluated according to the Modified Jadad Scale for randomized controlled trials (RCTs) [13] and the Newcastle-Ottawa Scale (NOS) for nonrandomized controlled trials [14].

\section{Statistical analysis}

The efficacy and safety of SWL, PCNL, and RIRS in the treatment of pediatric renal or ureteral calculi was accessed by the OR and mean difference with corresponding 95\% CI under the paired comparisons among the three treatment techniques. The OR value was calculated by using the $\mathrm{Z}$ test. In addition, if $p<0.05$, the difference was considered as statistically significant. When comparing the risk of continuous variables, the mean values and SDs are necessary for the pooled data. The random-effects model was used to generate the most conservative estimate. The chi-squared-based Q test was used to assess the heterogeneity with the significance level set to $p<0.10$. Subgroup analysis was conducted according to stone size $\leq 20 \mathrm{~mm}$ to increase the comparability of these procedures and reduce the heterogeneity of results. All of the statistical analyses were performed using the RevMan5.3 software.

\section{Results}

\section{Study identification and characteristics}

The process of the selection of studies included in this review is summarized in Fig. 1. In total, 13 articles were included in this quantitative synthesis [15-27]. There were 3 randomized controlled trials [17, 21, 24] (RCTs) (LE: 2b), 1 prospective case controlled study [16] (LE: $3 b$ ) and 9 retrospective case controlled studies [15, 18-20, 22, 23, 25-27] (LE: 3b) (Table 1). Seven nonrandomized studies were relatively high (NOS: 6 of 9 points) and 3 nonrandomized studies were medium (NOS: 5 of 9 points) in methodological quality. The methodological quality of the 3 RCTs were relatively low (Modified Jadad Scale: 2 of 3 points and 1 of 2 points) due to a lack allocation concealment and a lack blinding methods.

A total of 529 SWL cases (50.38\%), 241 PCNL cases (22.95\%), and 280 RIRS cases (26.67\%) were included. The baseline characteristics of the children are summarized in Table 2.

\section{Meta-analysis outcomes \\ Overall SFR and single-session SFR}

All the included studies defined stone free as no residual fragments except one study [22] that defined stone free as fragments no larger than $4 \mathrm{~mm}$. PCNL presented a significantly higher overall SFR (OR 2.69, 95\% CI 1.48 to $4.91, p=0.001$ ) and single-session SFR (OR 4.67, 95\% CI 1.68 to $12.98, p=0.003)$ than SWL. Similarly, the singlesession SFR of RIRS was significantly higher than SWL (OR 2.34, 95\% CI 1.21 to $4.54, p=0.01$ ), but there was no significant difference in the overall SFR between RIRS and SWL (OR 2.12, 95\% CI 1.00 to $4.48, p=0.05$ ). Furthermore, PCNL had similar effects in the overall SFR and single-session SFR in comparison with RIRS (OR 1.42, $95 \%$ CI 0.70 to $2.88, p=0.33$; OR $1.28,95 \%$ CI 0.71 to $2.32, p=0.41$ ). (Fig. 2).

\section{Complication rate, minor (Clavien-Dindo I-II) complication rate and major (Clavien-Dindo III-IV) complication rate}

Intriguingly, no significant difference in the complication rate was found among the three procedures (SWL vs PCNL OR $0.85,95 \%$ CI 0.25 to $2.89, p=0.79$; SWL vs RIRS OR $0.69,95 \%$ CI 0.28 to $1.66, p=0.40$; and RIRS vs PCNL OR $0.65,95 \%$ CI 0.32 to $1.30, p=0.22$ ). Furthermore, we analysed the postoperative complications according to grade. The results showed, similar to the overall complication rates, the minor (Clavien-Dindo I-II) complication rates (SWL vs PCNL OR $0.57,95 \%$ CI 0.20 to $1.57, p=0.27$; 


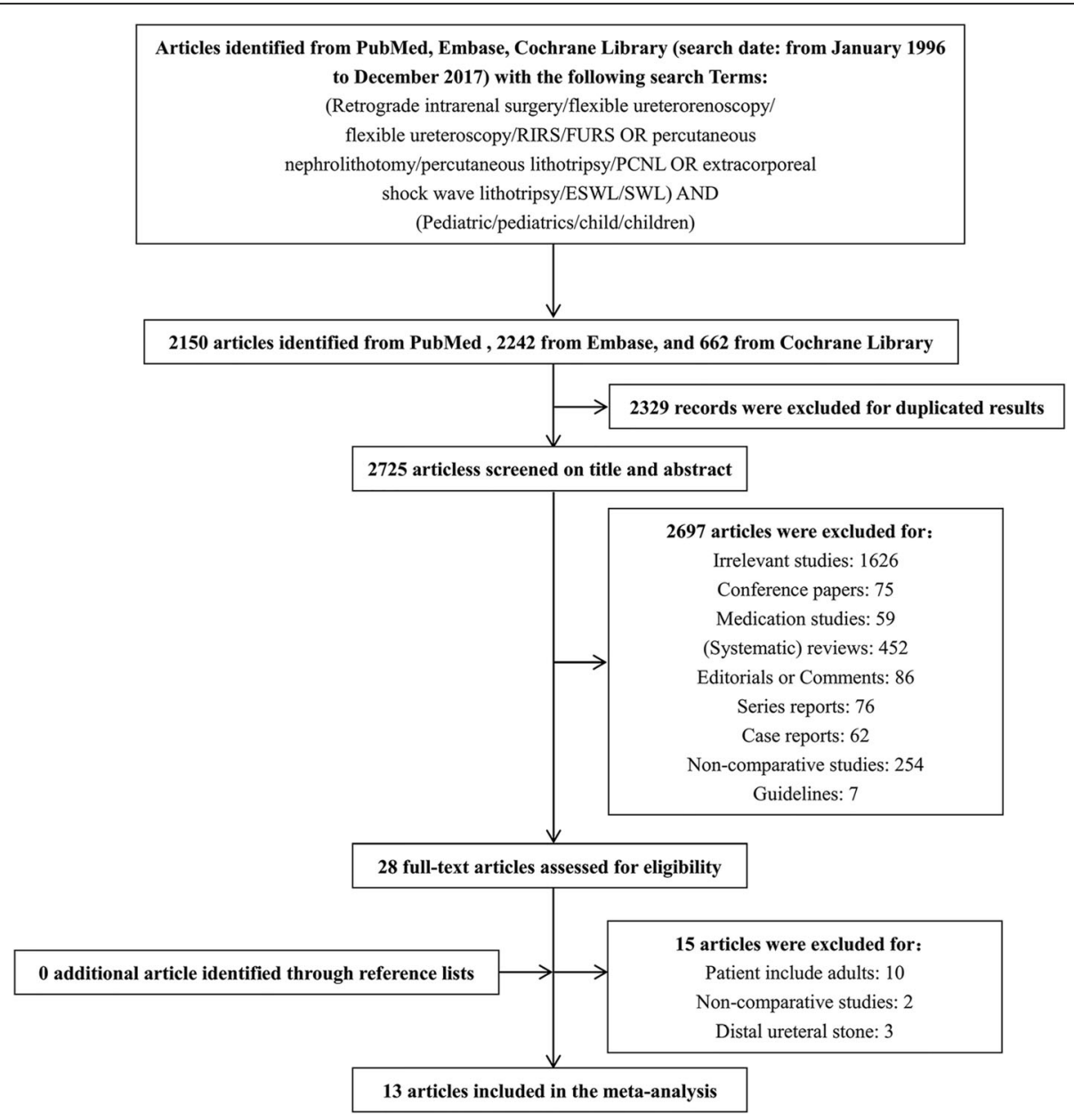

Fig. 1 PRISMA flow diagram of study selection

Table 1 Summary of publications included in the meta-analysis

\begin{tabular}{|c|c|c|c|c|c|c|c|c|}
\hline \multirow[t]{2}{*}{ Study } & \multirow[t]{2}{*}{ Study period } & \multirow[t]{2}{*}{ Country } & \multirow{2}{*}{$\begin{array}{l}\text { Study } \\
\text { design }\end{array}$} & \multicolumn{3}{|c|}{ Cases, $\mathrm{n}$} & \multirow[t]{2}{*}{ LE } & \multirow{2}{*}{$\begin{array}{l}\text { Study } \\
\text { quality }\end{array}$} \\
\hline & & & & $\overline{S W L}$ & PCNL & $\overline{\mathrm{RIRS}}$ & & \\
\hline Zeng et al [15] & $2005-2011$ & China & RCCS & 22 & 24 & - & $3 b$ & $6^{\mathrm{b}}$ \\
\hline Wadhwa et al [16] & 2005-2005 & India & PCCS & 8 & 6 & - & $3 b$ & $5^{\mathrm{b}}$ \\
\hline Kumar et al [17] & 2012-2013 & India & RCT & 106 & 106 & - & $2 b$ & $3^{\mathrm{a}}$ \\
\hline Hatipoglu et al [18] & 2010-2012 & Turkey & RCCS & 108 & 37 & - & $3 b$ & $6^{\mathrm{b}}$ \\
\hline Shokeir et al [19] & $1995-2004$ & Egypt & RCCS & 91 & 75 & - & $3 b$ & $6^{\mathrm{b}}$ \\
\hline ElSheemy et al [20] & 2010-2014 & Egypt & RCCS & 64 & 54 & - & $3 b$ & $6^{\mathrm{b}}$ \\
\hline Mokhless et al [21] & 3 months follow-up & Egypt & RCT & 30 & - & 30 & $2 b$ & $3^{\mathrm{a}}$ \\
\hline Freton et al [22] & 2000-2014 & France & RCCS & 100 & - & 46 & $3 b$ & $5^{\mathrm{b}}$ \\
\hline Resorlu et al [23] & 2008-2011 & Turkey & MRCCS & - & 106 & 95 & $3 b$ & $6^{\mathrm{b}}$ \\
\hline Saad et al [24] & $2011-2014$ & Egypt & $\mathrm{RCT}$ & - & 20 & 18 & $2 b$ & $2^{\mathrm{a}}$ \\
\hline Bas et al [25] & 2011-2015 & Turkey & RCCS & - & 45 & 36 & $3 b$ & $6^{\mathrm{b}}$ \\
\hline Sen et al [26] & $2015-2016$ & Turkey & RCCS & - & 25 & 23 & $3 b$ & $5^{\mathrm{b}}$ \\
\hline Pelit et al [27] & $2013-2016$ & Turkey & RCCS & - & 45 & 32 & $3 b$ & $6^{\mathrm{b}}$ \\
\hline
\end{tabular}

a.Modified Jadad scale (score from 0 to 7); b. Newcastle-Ottawa scale (score from 0 to 9). SWL Extracorporeal shockwave lithotripsy, PCNL Percutaneous nephrolithotomy, RIRS Retrograde intrarenal surgery, $L E$ Level of evidence, $R C C S$ Retrospective case control study, PCCS Prospective case control study, RCT Randomized control trail, MRCCS Multicenter retrospective case control study 
Table 2 Summary of pediatric patients' baseline of included studies

\begin{tabular}{|c|c|c|c|c|c|c|c|c|c|c|c|c|}
\hline \multirow[t]{3}{*}{ Author } & \multirow[t]{3}{*}{ Treatment } & \multirow{3}{*}{$\begin{array}{l}\text { Age, years } \\
\text { (Mean } \pm \text { SD) }\end{array}$} & \multicolumn{2}{|l|}{ Sex } & \multicolumn{5}{|c|}{ Stone location, $\mathrm{n}$ (units) } & \multirow{2}{*}{\multicolumn{2}{|c|}{ Side }} & \multirow{3}{*}{$\begin{array}{l}\text { Stone size, } \\
\mathrm{mm} \\
\text { (Mean } \pm \mathrm{SD})\end{array}$} \\
\hline & & & & & \multirow[t]{2}{*}{ Pelvis } & \multirow[t]{2}{*}{ Caliceal } & \multirow{2}{*}{$\begin{array}{l}\text { Pelvis + } \\
\text { caliceal }\end{array}$} & \multicolumn{2}{|c|}{ Staghorn stone } & & & \\
\hline & & & Male & Female & & & & Partial & Complete & Unilateral & Bilateral & \\
\hline \multirow[t]{2}{*}{ Zeng et al [15] } & SWL & $1.96 \pm 0.55$ & 17 & 5 & $5(5)$ & $\mathrm{O}(0)$ & $2(2)$ & $15(15)$ & $0(0)$ & 22 & 0 & $21.7 \pm 1.7$ \\
\hline & PCNL & $1.92 \pm 0.8$ & 15 & 9 & $6(7)$ & $0(0)$ & $3(4)$ & $12(12)$ & $3(3)$ & 24 & 1 & $21.4 \pm 3.5$ \\
\hline \multirow[t]{2}{*}{ Wadhwa et al [16] } & SWL & $3 \sim 12$ & 11 & 3 & $8(9)$ & $0(0)$ & $0(0)$ & $0(0)$ & $0(0)$ & NA & & $220.4^{a}$ \\
\hline & PCNL & 5.9 & & & $N A(4)$ & $0(0)$ & $\mathrm{NA}(4)$ & $1(1)$ & $0(0)$ & & & $1393.4^{\mathrm{a}}$ \\
\hline \multirow[t]{2}{*}{ Kumar et al [17] } & SWL & $10.7 \pm 1.3$ & 52 & 54 & NA & & & & & 106 & 0 & $12.9 \pm 1.3$ \\
\hline & PCNL & $10.3 \pm 1.2$ & 51 & 55 & & & & & & 106 & 0 & $12.7 \pm 1.2$ \\
\hline \multirow[t]{2}{*}{ Hatipoglu et al [18] } & SWL & $5.91 \pm 4.03$ & 61 & 47 & 50 & 58 & $0(0)$ & $0(0)$ & $0(0)$ & 108 & 0 & $11.32 \pm 2.84$ \\
\hline & PCNL & $8.43 \pm 4.84$ & 15 & 22 & 10 & 27 & $0(0)$ & $0(0)$ & $0(0)$ & 37 & 0 & $14.78 \pm 5.39$ \\
\hline \multirow[t]{2}{*}{ Shokeir et al [19] } & SWL & $6.4 \pm 1.4$ & 50 & 41 & $\mathrm{NA}(70)$ & $\mathrm{NA}(11)$ & $\mathrm{NA}(12)$ & $0(0)$ & $0(0)$ & 89 & 2 & $13.9 \pm 4.2$ \\
\hline & PCNL & $6.6 \pm 1.2$ & 45 & 30 & NA(52) & $\mathrm{NA}(14)$ & $\mathrm{NA}(16)$ & $0(0)$ & $0(0)$ & 68 & 7 & $14.4 \pm 3.1$ \\
\hline \multirow[t]{2}{*}{ ElSheemy et al [20] } & SWL & $4.06 \pm 0.96$ & 44 & 20 & 52 & 12 & $0(0)$ & $0(0)$ & $0(0)$ & 64 & 0 & $14.87 \pm 4.05$ \\
\hline & PCNL & $3.84 \pm 1.44$ & 33 & 21 & 39 & 15 & $0(0)$ & $0(0)$ & $0(0)$ & 54 & 0 & $15.98 \pm 4.31$ \\
\hline \multirow[t]{2}{*}{ Mokhless et al [21] } & SWL & $1 \sim 6$ & 40 & 20 & $15(\mathrm{NA})$ & $5(\mathrm{NA})$ & $10(N A)$ & $0(0)$ & $0(0)$ & 60 & 0 & $10-20$ \\
\hline & RIRS & $2.4 \pm 1.3$ & & & $17(\mathrm{NA})$ & $7(\mathrm{NA})$ & $6(\mathrm{NA})$ & $0(0)$ & $0(0)$ & & & $10-20$ \\
\hline \multirow[t]{2}{*}{ Freton et al [22] } & SWL & $6.7 \pm 0.6$ & NA & & Upper $\mathrm{t}$ & act urinary & stones (up & per uret & ar or kidney) & NA & & $19.5 \pm 1.5$ \\
\hline & RIRS & $9.1 \pm 0.9$ & & & & & & & & & & $21.6 \pm 2.0$ \\
\hline \multirow[t]{2}{*}{ Resorlu et al [23] } & PCNL & $9.6 \pm 4.9$ & 56 & 50 & $36(N A)$ & $70(N A)$ & $0(0)$ & $0(0)$ & $0(0)$ & & & $23.7 \pm 4.42$ \\
\hline & RIRS & $9.3 \pm 5.2$ & 53 & 42 & $29(N A)$ & $66(N A)$ & $0(0)$ & $0(0)$ & $0(0)$ & & & $14.3 \pm 3.81$ \\
\hline \multirow[t]{2}{*}{ Saad et al [24] } & PCNL & $6.93 \pm 3.55$ & 14 & 8 & $2(2)^{b}$ & & $17(17)^{c}$ & $3(3)$ & & 22 & 0 & $>20$ \\
\hline & RIRS & $6.44 \pm 4.84$ & 14 & 7 & $5(5)^{\mathrm{b}}$ & & $11(11)^{c}$ & $5(5)$ & & 21 & 0 & $>20$ \\
\hline \multirow[t]{2}{*}{ Bas et al [25] } & PCNL & $5.62 \pm 4.50$ & 23 & 22 & 27 & 18 & $0(0)$ & $0(0)$ & $0(0)$ & 45 & 0 & $13.97 \pm 3.46$ \\
\hline & RIRS & $8.39 \pm 4.72$ & 15 & 21 & 14 & 22 & $0(0)$ & $0(0)$ & $0(0)$ & 36 & 0 & $12.80 \pm 3.03$ \\
\hline \multirow[t]{2}{*}{ Sen et al [26] } & PCNL & $4 \pm 2.3$ & NA & & 1 & 24 & $0(0)$ & $0(0)$ & $0(0)$ & NA & & $12.2 \pm 2.8$ \\
\hline & RIRS & $10.9 \pm 3$ & & & 0 & 23 & $0(0)$ & $0(0)$ & $0(0)$ & & & $13.7 \pm 3.5$ \\
\hline \multirow[t]{2}{*}{ Pelit et al [27] } & PCNL & $3.71 \pm 1.89$ & 24 & 21 & \multirow{2}{*}{\multicolumn{5}{|c|}{ Renal stones }} & NA & & $21.06 \pm 5.61$ \\
\hline & RIRS & $3.65 \pm 1.95$ & 17 & 15 & & & & & & & & $19.30 \pm 4.21$ \\
\hline
\end{tabular}

a. mm2, b. single stone, c. multiple stones. SWL Extracorporeal shockwave lithotripsy, PCNL Percutaneous nephrolithotomy, RIRS Retrograde intrarenal surgery, NA Not available

SWL vs RIRS OR $0.98,95 \%$ CI 0.37 to $2.60, p=0.97$; and RIRS vs PCNL OR $0.69,95 \%$ CI 0.39 to $1.22, p=0.20$ ) and the major (Clavien-Dindo III-IV) complication rates (SWL vs PCNL OR $1.45,95 \%$ CI 0.49 to $4.31, p=0.28$; SWL vs RIRS OR $0.14,95 \%$ CI 0.01 to $1.43, p=0.10$; and RIRS vs PCNL OR 0.92 , 95\% CI 0.17 to $5.00, p=0.92$ ) were not significantly different among these three procedures (Fig. 3). The detailed complications reported by the included studies are summarized in Table 3.

\section{Operative time, fluoroscopy time and hospital stay}

Compared with the other two procedures, PCNL had a longer operative time, fluoroscopy time and hospital stay than SWL and RIRS (Fig. 4). In addition, SWL had a significantly shorter operative time (weighted mean difference [WMD] - 12.10, 95\% CI -15.16 to $-9.04, p<0.00001$ ) and hospital stay (WMD $-0.38,95 \% \mathrm{CI}-0.63$ to $-0.14, p=0.002$ ) than
RIRS. There was no significant difference in fluoroscopy time between SWL and RIRS (WMD 10.00, 95\% CI - 9.56 to 29.56, $p=0.32$ ). (Fig. 4).

\section{Retreatment rate and auxiliary procedure rate}

SWL exhibited a significantly higher retreatment rate than PCNL (OR 14.41, 95\% CI 8.41 to 24.71, $p<$ 0.00001 ) and RIRS (OR 26.95, 95\% CI 1.49 to 488.33 , $p=0.03$ ), while the retreatment rate between PCNL and RIRS (OR $0.55,95 \%$ CI 0.18 to $1.71, p=0.30$ ) did not show any significant difference. The auxiliary procedure rate of RIRS had no significant difference compared with the other two treatments. Furthermore, SWL had a significantly higher auxiliary procedure rate compared with PCNL (OR 2.78, 95\% CI 1.39 to 5.55, $p=0.004$ ) (Fig. 5). 


\section{Overall SFR}

\begin{tabular}{|c|c|c|c|c|c|c|c|c|c|c|}
\hline $\begin{array}{l}\text { a PCNL vs SWL } \\
\text { Study or Subgroup }\end{array}$ & $\begin{array}{r}\text { PCNL } \\
\text { Events }\end{array}$ & Total & $\begin{array}{l}\text { SWL } \\
\text { Events }\end{array}$ & Total & Weight & $\begin{array}{l}\text { Odds Ratio } \\
\text { M-H, Random, } 95 \% \mathrm{Cl} \\
\end{array}$ & & $\begin{array}{r}\text { Odds } \\
\text { M-H, Rand } \\
\end{array}$ & $\begin{array}{l}\text { Ratio } \\
\text { lom. } 95 \% \mathrm{Cl}\end{array}$ & \\
\hline EISheemy 2016 & 51 & 54 & 52 & 64 & $20.7 \%$ & $3.92[1.05,14.73]$ & & & & \\
\hline Hatipoglu 2013 & 33 & 37 & 95 & 108 & $25.6 \%$ & $1.13[0.34,3.71]$ & & & & \\
\hline Kumar 2015 & 100 & 106 & 88 & 106 & $38.6 \%$ & $3.41[1.30,8.97]$ & & & 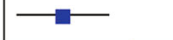 & \\
\hline Shokeir 2005 & 82 & 82 & 86 & 93 & $4.4 \%$ & $14.31[0.80,254.49]$ & & & & \\
\hline Wadhwa 2007 & 8 & 9 & 8 & 9 & $4.2 \%$ & $1.00[0.05,18.91]$ & & & & \\
\hline Zeng 2012 & 24 & 25 & 19 & 22 & $6.6 \%$ & $3.79[0.36,39.41]$ & & & & \\
\hline Total $(95 \% \mathrm{Cl})$ & & 313 & & 402 & $100.0 \%$ & $2.69[1.48,4.91]$ & & & & \\
\hline Total events & 298 & & 348 & & & & & & & \\
\hline $\begin{array}{l}\text { Heterogeneity: } \mathrm{Tau}^{2}=0 \\
\text { Test for overall effect: } 2\end{array}$ & $\begin{array}{l}0.00 ; \mathrm{Chi}^{2}= \\
\mathrm{z}=3.23(\mathrm{P}\end{array}$ & $\begin{array}{l}=4.48, \\
=0.00\end{array}$ & $\begin{array}{l}d f=5(P= \\
1)\end{array}$ & $=0.48)$ & $3 ; I^{2}=0 \%$ & & 0.01 & $\begin{array}{l}0.1 \\
\text { Favours SWL }\end{array}$ & $\begin{array}{l}10 \\
\text { Favours PCNL }\end{array}$ & 100 \\
\hline $\begin{array}{l}\text { b RIRS vs SWL } \\
\text { Study or Subgroup }\end{array}$ & $\begin{array}{l}\text { RIRS } \\
\text { Events }\end{array}$ & Total & $\begin{array}{l}\text { SWL } \\
\text { Events }\end{array}$ & & Weight & $\begin{array}{c}\text { Odds Ratio } \\
\text { M-H. Random, } 95 \% \mathrm{Cl}\end{array}$ & & $\begin{array}{r}\text { Odds } \\
\text { M-H, Rand }\end{array}$ & $\begin{array}{l}\text { Ratio } \\
\text { dom. } 95 \% \mathrm{Cl}\end{array}$ & \\
\hline Freton 2016 & 35 & 46 & 60 & 100 & $90.7 \%$ & $2.12[0.97,4.66]$ & & & D - & \\
\hline Mokhless 2014 & 29 & 30 & 28 & 30 & $9.3 \%$ & $2.07[0.18,24.15]$ & & & & \\
\hline Total $(95 \% \mathrm{Cl})$ & & 76 & & 130 & $100.0 \%$ & $2.12[1.00,4.48]$ & & & & \\
\hline Total events & 64 & & 88 & & & & & & & \\
\hline $\begin{array}{l}\text { Heterogeneity: } \mathrm{Tau}^{2}= \\
\text { Test for overall effect: }\end{array}$ & $\begin{array}{l}0.00 ; \mathrm{Chi}^{2}= \\
\mathrm{z}=1.96(\mathrm{P}\end{array}$ & $\begin{array}{l}=0.00, \\
=0.05\end{array}$ & $d f=1(P$ & $=0.99$ & 9); $1^{2}=0 \%$ & & 0.01 & $\begin{array}{l}0.1 \\
\text { Favours SWL }\end{array}$ & $\begin{array}{l}10 \\
\text { Favours RIRS }\end{array}$ & 100 \\
\hline $\begin{array}{l}\text { C PCNL vs RIRS } \\
\text { Study or Subgroup }\end{array}$ & $\begin{array}{r}\text { PCNL } \\
\text { Events } \\
\end{array}$ & Total & $\begin{array}{r}\text { RIRS } \\
\text { Events }\end{array}$ & Total & Weight & $\begin{array}{l}\text { Odds Ratio } \\
\text { M-H. Random. } 95 \% \mathrm{Cl}\end{array}$ & & $\begin{array}{r}\text { Odds } \\
\text { M-H, Rand } \\
\end{array}$ & $\begin{array}{l}\text { Ratio } \\
\text { dom, } 95 \% \mathrm{Cl}\end{array}$ & \\
\hline Bas 2016 & 36 & 45 & 31 & 36 & $25.5 \%$ & $0.65[0.20,2.13]$ & & $\longrightarrow$ & & \\
\hline Pelit 2016 & 41 & 45 & 29 & 32 & $16.6 \%$ & $1.06[0.22,5.10]$ & & & & \\
\hline Resorlu 2012 & 100 & 106 & 84 & 95 & $31.1 \%$ & $2.18[0.77,6.15]$ & & & & \\
\hline Saad 2015 & 21 & 22 & 15 & 21 & $9.2 \%$ & $8.40[0.91,77.21]$ & & & & \\
\hline Sen 2017 & 21 & 25 & 19 & 23 & $17.6 \%$ & $1.11[0.24,5.05]$ & & & & \\
\hline Total $(95 \% \mathrm{Cl})$ & & 243 & & 207 & $100.0 \%$ & $1.42[0.70,2.88]$ & & & & \\
\hline Total events & 219 & & 178 & & & & & & & \\
\hline $\begin{array}{l}\text { Heterogeneity: } \operatorname{Tau}^{2}=0 \\
\text { Test for overall effect: }\end{array}$ & $\begin{array}{l}0.14 ; \mathrm{Ch}^{2}= \\
\mathrm{z}=0.98(\mathrm{P}\end{array}$ & $\begin{array}{l}=5.07, \\
=0.33\end{array}$ & $\mathrm{df}=4(\mathrm{P}$ & $=0.28$ & B); $1^{2}=21 \%$ & & 0.01 & $\begin{array}{l}0.1 \\
\text { Favours RIRS }\end{array}$ & $\begin{array}{l}10 \\
\text { Favours PCNL }\end{array}$ & 100 \\
\hline
\end{tabular}

\section{Single-session SFR}

\begin{tabular}{|c|c|c|c|c|c|c|c|c|c|c|}
\hline $\begin{array}{l}\text { d PCNL vs SWL } \\
\text { Study or Subgroup }\end{array}$ & $\begin{array}{r}\text { PCNL } \\
\text { Events }\end{array}$ & Total & $\begin{array}{c}\text { SWL } \\
\text { Events }\end{array}$ & & Weight & $\begin{array}{l}\text { Odds Ratio } \\
\text { M-H, Random, } 95 \% \mathrm{Cl}\end{array}$ & & $\begin{array}{l}\text { Odds } \mathrm{F} \\
\text { M-H. Rando }\end{array}$ & $\begin{array}{l}\text { Ratio } \\
\text { om. } 95 \% \mathrm{Cl}\end{array}$ & \\
\hline EISheemy 2016 & 48 & 54 & 28 & 64 & $26.6 \%$ & $10.29[3.85,27.46]$ & & & $=$ & \\
\hline Hatipoglu 2013 & 29 & 37 & 77 & 108 & $27.8 \%$ & $1.46[0.60,3.54]$ & & & & \\
\hline Shokeir 2005 & 78 & 82 & 79 & 93 & $24.3 \%$ & $3.46[1.09,10.96]$ & & & & \\
\hline Zeng 2012 & 21 & 25 & 7 & 22 & $21.3 \%$ & $11.25[2.79,45.43]$ & & & & \\
\hline Total $(95 \% \mathrm{Cl})$ & & 198 & & 287 & $100.0 \%$ & $4.67[1.68,12.98]$ & & & & \\
\hline Total events & 176 & & 191 & & & & & & & \\
\hline $\begin{array}{l}\text { Heterogeneity: } \mathrm{Tau}^{2}= \\
\text { Test for overall effect: }\end{array}$ & $\begin{array}{l}0.77 ; \mathrm{Ch}^{2}= \\
\mathrm{Z}=2.95(\mathrm{P}\end{array}$ & $\begin{array}{l}=10.68 \\
=0.00\end{array}$ & $\begin{array}{l}\text {, df }=3(P \\
3)\end{array}$ & $P=0.0$ & 11); $\left.\right|^{2}=72$ & & 0.01 & $\begin{array}{c}0.1 \\
\text { Favours SWL }\end{array}$ & $\begin{array}{c}10 \\
\text { Favours PCNL }\end{array}$ & 100 \\
\hline $\begin{array}{l}\text { C RIRS vs SWL } \\
\text { Study or Subgroup }\end{array}$ & $\begin{array}{c}\text { RIRS } \\
\text { Events }\end{array}$ & Total & $\begin{array}{c}\text { SWL } \\
\text { Events }\end{array}$ & Total & Weight & $\begin{array}{c}\text { Odds Ratio } \\
\text { M-H, Random, } 95 \% \mathrm{Cl}\end{array}$ & & $\begin{array}{r}\text { Odds } \\
\text { M-H, Rando } \\
\end{array}$ & tio & \\
\hline Freton 2016 & 17 & 46 & 21 & 100 & $74.4 \%$ & $2.21[1.02,4.75]$ & & & & \\
\hline Mokhless 2014 & 26 & 30 & 21 & 30 & $25.6 \%$ & $2.79[0.75,10.33]$ & & & & \\
\hline Total $(95 \% \mathrm{Cl})$ & & 76 & & 130 & $100.0 \%$ & $2.34[1.21,4.54]$ & & & & \\
\hline Total events & 43 & & 42 & & & & & & & \\
\hline $\begin{array}{l}\text { Heterogeneity: } \mathrm{Tau}^{2}=0 \\
\text { Test for overall effect: }\end{array}$ & $\begin{array}{l}0.00 ; \mathrm{Chi}^{2}= \\
\mathrm{Z}=2.52(\mathrm{P}\end{array}$ & $\begin{array}{l}=0.09 \\
=0.01\end{array}$ & $d f=1(P=$ & $=0.76)$ & 6); $1^{2}=0 \%$ & & 0.01 & $\begin{array}{l}0.1 \\
\text { Favours SWL }\end{array}$ & $\begin{array}{c}10 \\
\text { Favours RIRS }\end{array}$ & 100 \\
\hline $\begin{array}{l}\text { f PCNL vs RIRS } \\
\text { Study or Subgroup }\end{array}$ & $\begin{array}{r}\text { PCNL } \\
\text { Events }\end{array}$ & Total & $\begin{array}{c}\text { RIRS } \\
\text { Events }\end{array}$ & Total & Weight & $\begin{array}{l}\text { Odds Ratio } \\
\text { M-H. Random, } 95 \% \mathrm{Cl}\end{array}$ & & $\begin{array}{r}\text { Odds } \mathrm{I} \\
\mathrm{M}-\mathrm{H}, \text { Rando }\end{array}$ & $\begin{array}{l}\text { Ratio } \\
\text { om. } 95 \% \mathrm{Cl}\end{array}$ & \\
\hline Pelit 2016 & 38 & 45 & 24 & 32 & $27.0 \%$ & $1.81[0.58,5.63]$ & & & & \\
\hline Resorlu 2012 & 91 & 106 & 80 & 95 & $57.9 \%$ & $1.14[0.52,2.47]$ & & & & \\
\hline Sen 2017 & 21 & 25 & 19 & 23 & $15.1 \%$ & $1.11[0.24,5.05]$ & & & & \\
\hline Total $(95 \% \mathrm{Cl})$ & & 176 & & 150 & $100.0 \%$ & $1.28[0.71,2.32]$ & & & & \\
\hline Total events & 150 & & 123 & & & & & & & \\
\hline $\begin{array}{l}\text { Heterogeneity: } \operatorname{Tau}^{2}= \\
\text { Test for overall effect: }\end{array}$ & $\begin{array}{l}0.00 ; \mathrm{Chi}^{2}= \\
\mathrm{z}=0.83(\mathrm{P}\end{array}$ & $\begin{array}{l}=0.48 \\
=0.41\end{array}$ & $d f=2(P=$ & $=0.79)$ & 9); $1^{2}=0 \%$ & & 0.01 & $\begin{array}{l}0.1 \\
\text { Favours RIRS }\end{array}$ & $\begin{array}{c}10 \\
\text { PCNL }\end{array}$ & 100 \\
\hline
\end{tabular}

Fig. 2 Forest plot comparing overall SFR between (a) PCNL and SWL, b RIRS and SWL, c PCNL and RIRS, and single-session SFR between (d) PCNL and SWL, e RIRS and SWL, f PCNL and RIRS 


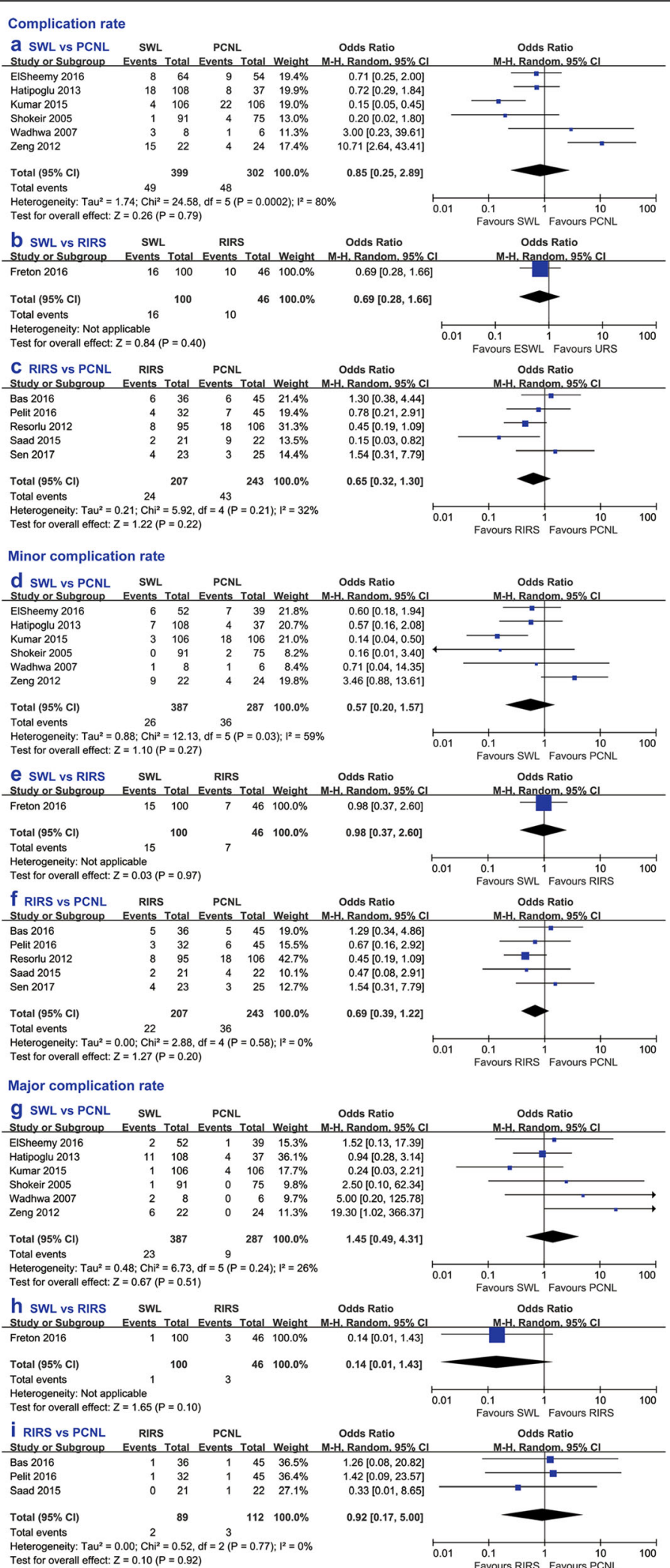

Fig. 3 Forest plot comparing complication rate between (a) SWL and PCNL, b SWL and RIRS, c RIRS and PCNL, minor complication rate between (d) SWL and PCNL, e SWL and RIRS, f RIRS and PCNL, and major complication rate between (g) SWL and PCNL, h SWL and RIRS, i RIRS and PCNL 
Table 3 Summary of detailed complications of included studies

\begin{tabular}{|c|c|c|c|c|c|c|c|c|c|c|c|c|}
\hline \multirow[t]{2}{*}{ Author } & \multirow[t]{2}{*}{ Treatment } & \multicolumn{11}{|c|}{ Complications, n(\%) } \\
\hline & & Total & $\begin{array}{l}\text { Renal } \\
\text { colic }\end{array}$ & Fever & $\begin{array}{l}\text { Urinary } \\
\text { infection }\end{array}$ & Hematuria & Uroepsis & Steinstrasse & $\begin{array}{l}\text { Blood } \\
\text { transfusion }\end{array}$ & $\begin{array}{l}\text { Ureteral } \\
\text { injury }\end{array}$ & Perforation & Others \\
\hline \multirow{2}{*}{$\begin{array}{l}\text { Zeng et al } \\
{[15]}\end{array}$} & SWL & 15(68.19) & $5(22.73)$ & $4(18.19)$ & - & $1(4.55)$ & - & $4(18.19)$ & - & - & - & $1(4.55)$ \\
\hline & PCNL & $4(16)$ & - & $4(16)$ & - & - & - & - & - & - & - & - \\
\hline \multirow{2}{*}{$\begin{array}{l}\text { Wadhwa } \\
\text { et al [16] }\end{array}$} & SWL & $3(37.5)$ & - & $1(12.5)$ & - & - & - & $2(25)$ & - & - & - & - \\
\hline & PCNL & $1(16.7)$ & - & $1(16.7)$ & - & - & - & - & - & - & - & - \\
\hline \multirow{2}{*}{$\begin{array}{l}\text { Kumar et al } \\
{[17]}\end{array}$} & SWL & $4(3.7)$ & $2(1.85)$ & - & $1(0.93)$ & $1(0.93)$ & - & - & - & - & - & - \\
\hline & PCNL & $22(20.75)$ & $5(4.72)$ & - & $9(8.49)$ & $8(7.55)$ & - & - & - & - & - & - \\
\hline \multirow{2}{*}{$\begin{array}{l}\text { Hatipoglu } \\
\text { et al [18] }\end{array}$} & SWL & $18(16.67)$ & $7(6.6)$ & - & - & - & - & $11(10.38)$ & - & - & - & - \\
\hline & PCNL & $8(21.62)$ & $4(10.81)$ & - & - & - & - & $1(2.70)$ & - & - & - & $3(8.11)$ \\
\hline \multirow{2}{*}{$\begin{array}{l}\text { Shokeir et al } \\
\text { [19] }\end{array}$} & SWL & $1(1.1)$ & - & - & - & - & - & $1(1.1)$ & - & - & - & - \\
\hline & PCNL & $4(5.41)$ & - & $2(2.7)$ & - & - & - & - & $1(1.35)$ & - & $1(1.35)$ & - \\
\hline \multirow{2}{*}{$\begin{array}{l}\text { ElSheemy } \\
\text { et al [20] }\end{array}$} & SWL & $8(15.4)$ & - & $4(7.7)$ & $4(7.7)$ & - & - & $4(7.7)$ & - & - & - & - \\
\hline & PCNL & $8(20.5)$ & - & $7(17.94)$ & $2(5.1)$ & - & - & - & - & - & $1(2.6)$ & $2(5.1)$ \\
\hline \multirow{2}{*}{$\begin{array}{l}\text { Mokhless } \\
\text { et al [21] }\end{array}$} & SWL & $N A^{a}$ & - & - & - & - & - & - & - & - & - & - \\
\hline & RIRS & $N A^{a}$ & - & - & - & - & - & - & - & - & - & - \\
\hline \multirow{2}{*}{$\begin{array}{l}\text { Freton et al } \\
\text { [22] }\end{array}$} & SWL & $16(16)$ & $11(11)$ & $1(1)$ & $2(2)$ & - & - & - & - & - & - & $2(2)$ \\
\hline & RIRS & $10(21.7)$ & - & - & $3(6.5)$ & $1(2.2)$ & - & $1(2.2)$ & - & - & - & $5(10.9)$ \\
\hline \multirow{2}{*}{$\begin{array}{l}\text { Resorlu et al } \\
\text { [23] }\end{array}$} & PCNL & $18(17)^{b}$ & - & - & - & - & - & - & - & - & - & - \\
\hline & RIRS & $8(8.4)^{b}$ & - & - & - & - & - & - & - & - & - & - \\
\hline \multirow{2}{*}{$\begin{array}{l}\text { Saad et al } \\
{[24]}\end{array}$} & PCNL & $9(40.9)$ & - & $4(18.2)$ & - & - & - & - & $3(13.6)$ & - & - & $2(9.1)$ \\
\hline & RIRS & $2(9.5)$ & - & $2(9.5)$ & - & - & - & - & - & - & - & - \\
\hline \multirow[t]{2}{*}{ Bas et al [25] } & PCNL & $6(13.3)$ & $4(8.9)$ & $1(2.2)$ & $1(2.2)$ & - & - & - & - & - & - & - \\
\hline & RIRS & $6(16.7)$ & 2(5.6) & $2(5.6)$ & $2(5.6)$ & - & - & - & - & - & - & - \\
\hline \multirow[t]{2}{*}{ Sen et al [26] } & PCNL & $3(12)$ & $3(12)$ & $2(8)$ & - & - & - & - & - & - & - & - \\
\hline & RIRS & $4(17.3)$ & $4(17.3)$ & $4(17.3)$ & - & - & $1(4.3)$ & - & - & - & - & - \\
\hline \multirow{2}{*}{$\begin{array}{l}\text { Pelit et al } \\
{[27]}\end{array}$} & PCNL & $7(15.5)$ & - & - & $2(4.4)$ & - & - & - & $3(6.7)$ & - & $1(2.2)$ & $1(2.2)$ \\
\hline & RIRS & $4(12.5)$ & - & - & $3(9.4)$ & - & - & - & - & 1(3.1) & - & - \\
\hline
\end{tabular}

a. No major complications; b. No detailed complications. SWL Extracorporeal shockwave lithotripsy, PCNL Percutaneous nephrolithotomy, RIRS Retrograde intrarenal surgery

EQ

According to the calculated EQs (Fig. 6), PCNL had a higher efficiency than SWL (OR 5.49, 95\% CI 3.73 to $8.06, p<0.00001$ ) and RIRS (OR 8.14, 95\% CI 4.75 to $13.98, p<0.00001)$. Only one study [20], which compared RIRS to SWL, provided enough data to identify a lower EQ of RIRS than SWL (OR 0.32, 95\% CI 0.11 to 0.94, $p=0.04$ ).

\section{Subgroup analyses PCNL and SWL}

Obviously, PCNL still presented a significantly higher overall SFR (OR 3.94, 95\% CI 1.58 to 9.86, $p=0.003$ ) and single-session SFR (OR 5.46, 95\% CI 2.34 to 12.73 , $p<0.0001)$ than SWL in the treatment of pediatric patients with upper urinary tract stone of $\leq 20 \mathrm{~mm}$ (Fig. 7). Except for the approximate major complication rate (OR
1.74, 95\% CI 0.19 to $15.80, p=0.62$ ), PCNL had both a significantly higher complication rate (OR 6.32, 95\% CI 2.35 to $16.98, p=0.0003)$ and a higher minor complication rate (OR 6.90, 95\% CI 2.16 to $22.03, p=0.001$ ) (Fig. 7). However, pediatric patients with a stone size $\leq 20 \mathrm{~mm}$ who were treated with SWL were more likely to have multiple operations (PCNL vs SWL: OR 0.07, 95\% CI 0.04 to $0.13, p<0.00001)$ and additional procedures (PCNL vs SWL: OR $0.43,95 \%$ CI 0.20 to $0.92, p=0.03$ ) (Fig. 7).

\section{RIRS and SWL}

RIRS had a significantly higher single-session SFR than SWL (OR 3.16, 95\% CI 1.21 to 8.28, $p=0.02$ ) (Fig. 8). Although SWL was always conducted as an outpatient procedure, the results of the subgroup analyses did not show statistically significant differences of hospital stay 


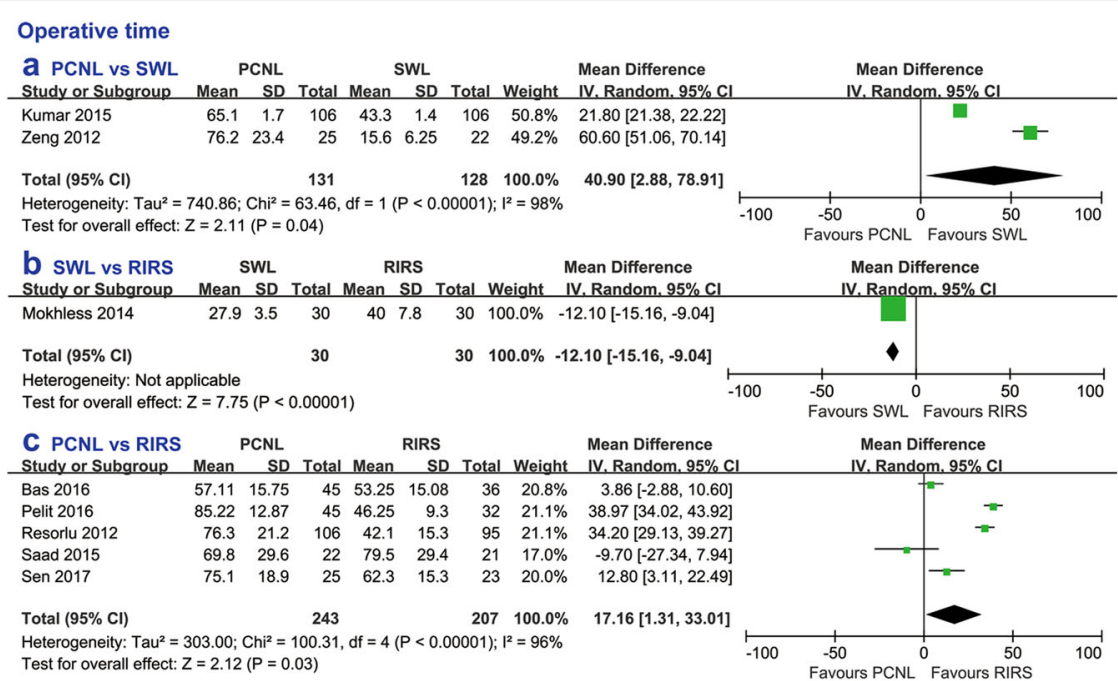

Fluoroscopy time

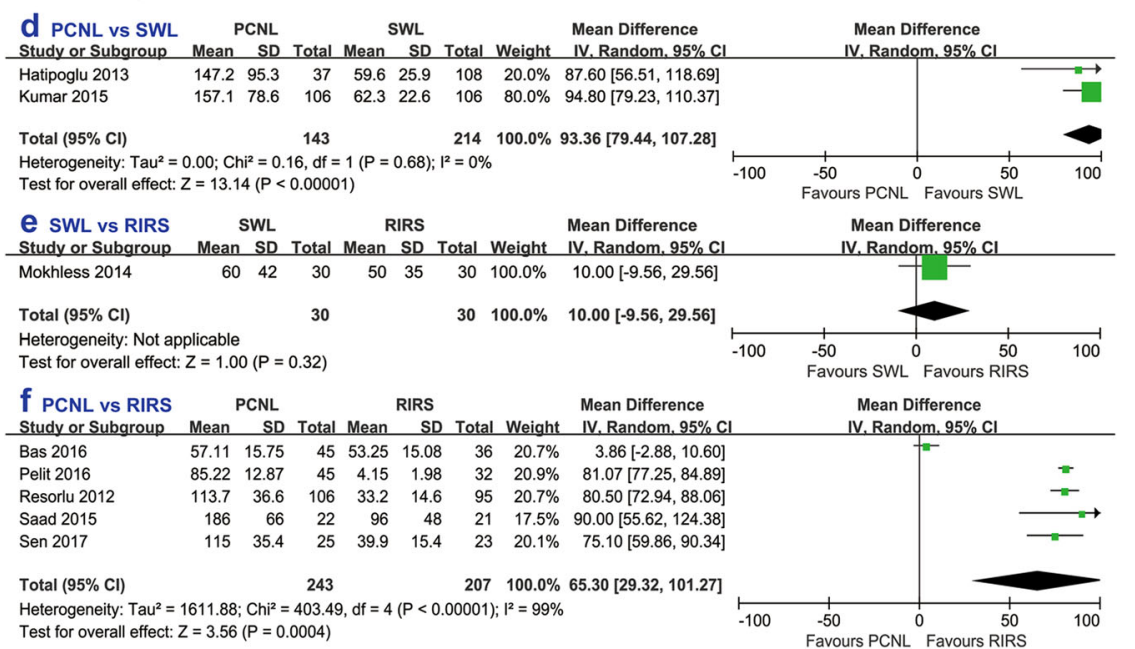
Test for overall effect: $Z=3.56(P=0.0004)$

Hospital stay

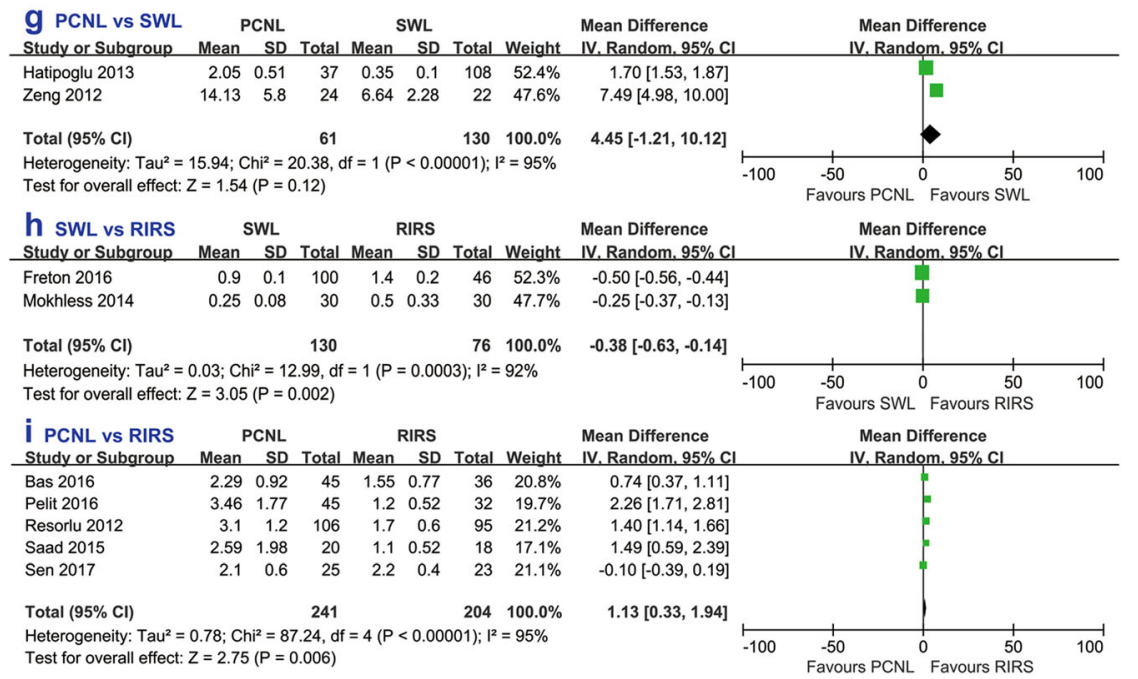

Fig. 4 Forest plot comparing operative time between (a) PCNL and SWL, b SWL and RIRS, c PCNL and RIRS, fluoroscopy time between (d) PCNL and SWL, e SWL and RIRS, f PCNL and RIRS, and hospital stay between (g) PCNL and SWL, h SWL and RIRS, i PCNL and RIRS 
Retreatment rate

\begin{tabular}{lrrrrr}
$\begin{array}{l}\text { a SWL vs PCNL } \\
\text { Study or Subgroup }\end{array}$ & \multicolumn{1}{c}{ SWL } & \multicolumn{4}{c}{ PCNL } \\
Events & Total & Events & Total & Weigh \\
\hline ElSheemy 2016 & 32 & 64 & 4 & 54 & $22.8 \%$ \\
Hatipoglu 2013 & 37 & 108 & 0 & 37 & $3.7 \%$ \\
Kumar 2015 & 44 & 106 & 3 & 106 & $19.8 \%$ \\
Shokeir 2005 & 51 & 91 & 7 & 75 & $37.4 \%$ \\
Wadhwa 2007 & 7 & 9 & 0 & 9 & $2.9 \%$ \\
Zeng 2012 & 11 & 22 & 3 & 25 & $13.5 \%$ \\
& & & & & \\
Total (95\% Cl) & & 400 & & 306 & $100.0 \%$ \\
Total events & 182 & & 17 & &
\end{tabular}

Heterogeneity: $\mathrm{Tau}^{2}=0.00 ; \mathrm{Chi}^{2}=3.05, \mathrm{df}=5(P=0.69) ; \mathrm{I}^{2}=0 \%$

Test for overall effect: $Z=9.70(P<0.00001)$

Odds Ratio Odds Ratio

-H. Random, $95 \% \mathrm{Cl}$

$12.50[4.04,38.70]$

$39.34[2.35,658.66]$

$24.37[7.26,81.81]$

$12.39[5.13,29.90]$

$57.00[2.36,1375.77]$

$7.33[1.69,31.81]$

$14.41[8.41,24.71]$

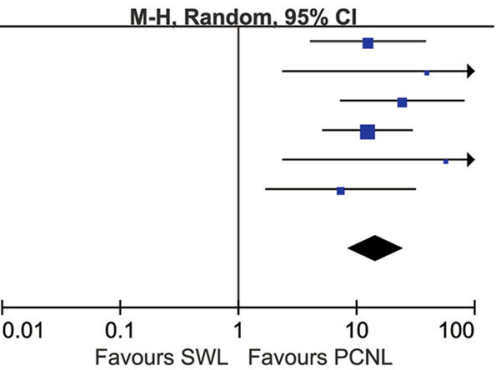

Odds Ratio

b sWL vs RIRS SWL RIRS

Events Total Events Total Weight M-H, Random, 95\% Cl

\begin{tabular}{lrrrrrr} 
Study or Subgroup & Events & Total & Events & Total & Weight & M-H, Random, 95\% C \\
\hline Mokhless 2014 & 9 & 30 & 0 & 30 & $100.0 \%$ & $26.95[1.49,488.33]$
\end{tabular}

Total $(95 \% \mathrm{Cl})$

30

$30100.0 \%$

$26.95[1.49,488.33]$

Total events

90

Heterogeneity: Not applicable

Test for overall effect: $Z=2.23(P=0.03)$

C PCNL vs RIRS PCNL 0

$\begin{array}{lrrrrr}\text { Pelit 2016 } & 0 & 45 & 3 & 32 & 14.4 \% \\ \text { Resorlu 2012 } & 4 & 106 & 4 & 95 & 64.7 \% \\ \text { Saad 2015 } & 1 & 20 & 2 & 18 & 20.9 \%\end{array}$

Saad 2015

Total $(95 \% \mathrm{Cl})$

171

$145 \quad 100.0 \%$

Total events $5 \quad 9$

Heterogeneity: $\mathrm{Tau}^{2}=0.00 ; \mathrm{Chi}^{2}=1.91, \mathrm{df}=2(\mathrm{P}=0.39) ; \mathrm{I}^{2}=0 \%$

Test for overall effect: $Z=1.03(P=0.30)$

Odds Ratio

M-H, Random, $95 \% \mathrm{Cl}$

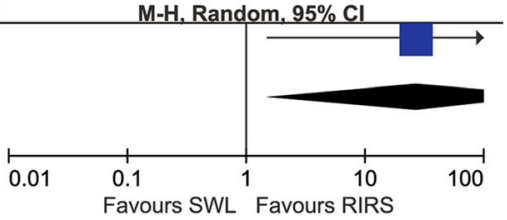

Odds Ratio

Odds Ratio

M-H, Random, $95 \% \mathrm{Cl}$ M-H, Random, $95 \% \mathrm{Cl}$

$0.09[0.00,1.86]$
$0.89[0.22,3.67]$
$0.42[0.03,5.08]$

$0.55[0.18,1.72]$

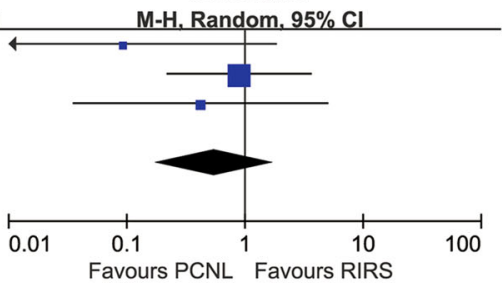

Auxiliary procedure rate

d SWL vS PCNL SWL

PCNL

Odds Ratio

Odds Ratio

Study or Subgroup Events Total Events Total Weight M-H, Random, $95 \% \mathrm{Cl}$

$\begin{array}{llllll}\text { EISheemy } 2016 & 6 & 52 & 1 & 39 & 10.3 \%\end{array}$

Kumar 2015

Shokeir 2005

Wadhwa 2007

Zeng 2012

15106

$\begin{array}{lll}6 & 106 & 49.1 \%\end{array}$

$\begin{array}{lll}4 & 82 & 31.2 \%\end{array}$

893

$\begin{array}{rrr}4 & 82 & 31.2 \% \\ 0 & 9 & 4.9 \%\end{array}$

$\begin{array}{lrr}0 & 9 & 4.9 \% \\ 0 & 25 & 4.5 \%\end{array}$

$4.96[0.57,42.99]$

$2.75[1.02,7.38]$

$1.84[0.53,6.34]$

$10.23[0.45,233.23]$

$3.56[0.14,91.92]$

Total $(95 \% \mathrm{Cl})$

282

$261100.0 \%$

Total events

$33 \quad 11$

Heterogeneity: $\mathrm{Tau}^{2}=0.00 ; \mathrm{Chi}^{2}=1.41, \mathrm{df}=4(\mathrm{P}=0.84) ; \mathrm{I}^{2}=0 \%$

Test for overall effect: $Z=2.89(P=0.004)$

e SWL vs RIRS SWL

RIRS

$2.78[1.39,5.55]$

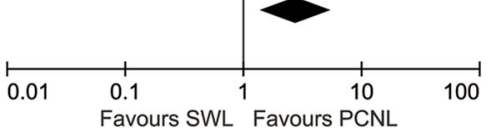

Odds Ratio

Study or Subgroup Events Total Events Total Weight M-H, Random, 95\% Cl

$\begin{array}{llllll}\text { Mokhless } 2014 & 0 & 30 & 1 & 30 & 100.0 \%\end{array}$

$\begin{array}{llll}\text { Total }(95 \% \mathrm{Cl}) & 30 & 30 & 100.0 \%\end{array}$

$\begin{array}{lll}\text { Total events } & 0 & 1\end{array}$

Heterogeneity: Not applicable

Test for overall effect: $Z=0.68(P=0.49)$

f PCNL vS RIRS PCNL

PCNL RIRS

$0.32[0.01,8.24]$

$0.32[0.01,8.24]$

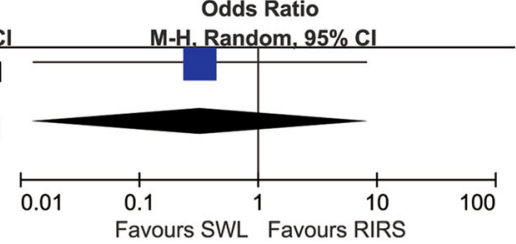

Odds Ratio

Odds Ratio

Study or Subgroup Events Total Events Total Weight M-H, Random, 95\% Cl

Pelit 2016

Resorlu 2012

Saad 2015

$\begin{array}{lllll}5 & 45 & 2 & 32 & 32.9 \%\end{array}$

$1.88[0.34,10.33]$

$1.36[0.37,4.99]$

$0.17[0.01,3.83]$

Total $(95 \% \mathrm{Cl})$

173

Total events

11

$148 \quad 100.0 \%$

Heterogeneity: $\mathrm{Tau}^{2}=0.00 ; \mathrm{Chi}^{2}=1.82, \mathrm{df}=2(\mathrm{P}=0.40) ; \mathrm{I}^{2}=0 \%$

Test for overall effect: $Z=0.42(P=0.68)$

$1.23[0.46,3.28]$

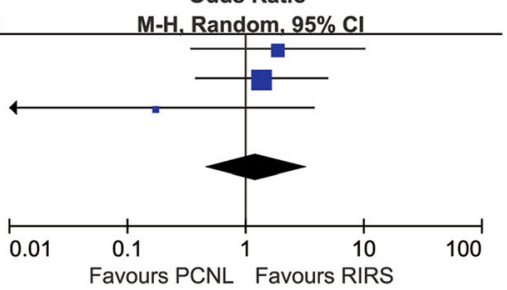

Fig. 5 Forest plot comparing retreatment rate between (a) SWL and PCNL, b SWL and RIRS, c RIRS and PCNL, and auxiliary procedure rate between (d) SWL and PCNL, e SWL and RIRS, f RIRS and PCNL 


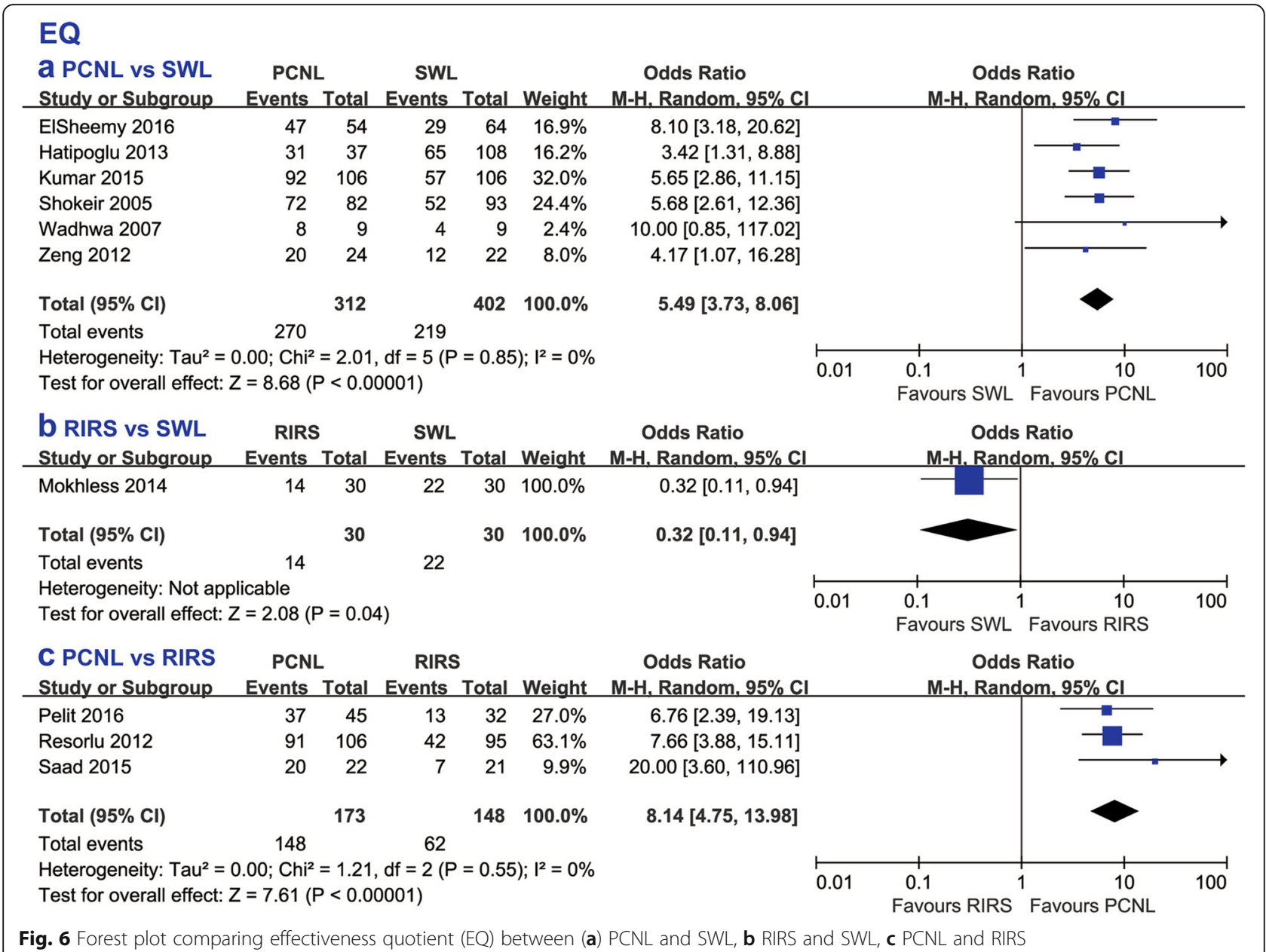

between RIRS and SWL (WMD 0.57, 95\% CI -0.06 to 1.21, $p=0.08$ ) (Fig. 8).

\section{PCNL and RIRS}

Overall SFR, single-session SFR, complication rate, minor complication rate, operative time, fluoroscopy time and hospital stay were all not significantly different between PCNL and RIRS (Fig. 9).

\section{Discussion}

Pediatric urolithiasis, which has a high risk of a relapse, can currently be managed by SWL, PCNL, RIRS, and laparoscopic or open surgery. Regardless of the selection of treatment modalities, the final objective is to render the pediatric patient stone free and to reduce the recurrence risk to a minimum. The results from this systematic review illustrated that PCNL presented a significantly higher overall SFR (OR 2.69, 95\% CI 1.48 to $4.91, p=0.001$ ), higher single-session SFR (OR 4.67, 95\% CI 1.68 to 12.98 , $p=0.003$ ), and higher EQ. (OR 5.49, 95\% CI 3.73 to 8.06, $p<0.00001)$ compared with SWL. The RCT of SWL vs PCNL conducted by Kumar A, et al. [17] proved that
PCNL was more efficient for stone sizes of $10-20 \mathrm{~mm}$ than SWL (EQ: $86.96 \%$ vs $53.33 \%$ ), and the identical conclusion was also reached by the retrospective case controlled studies. Although the pooled results demonstrated the overall SFR and single-session SFR of PCNL were both significantly higher than SWL, there was no significant difference in several studies in terms of the overall SFR (PCNL vs SWL: $96 \%$ vs $86.3 \%$; $88 \%$ vs $88 \% ; 89.2 \%$ vs $88 \%$; $94.9 \%$ vs $84.6 \%)[15,16,18,20]$. Considering the lower retreatment rate and auxiliary procedure rate, these studies claimed that PCNL is a feasible and more efficient treatment for pediatric renal stones.

The pooled results revealed there was no significant difference in the SFR between PCNL and RIRS regardless of the stone size of the pediatric patients. Although Resorlu B, et al. [23] also reported that no significant difference of the SFR was found between PCNL and RIRS (94.3\% vs $92.6 \%$ ), if dividing patients into subgroups according to stone size, PCNL was apparently superior to RIRS (> 20 mm: $83.9 \%$ vs $50 \%$; < 20 mm: $100 \%$ vs $87.3 \%$ ). Nevertheless, the length of hospital stay was significantly shorter in RIRS pediatric patients in comparison with 


\section{PCNL vs SWL}

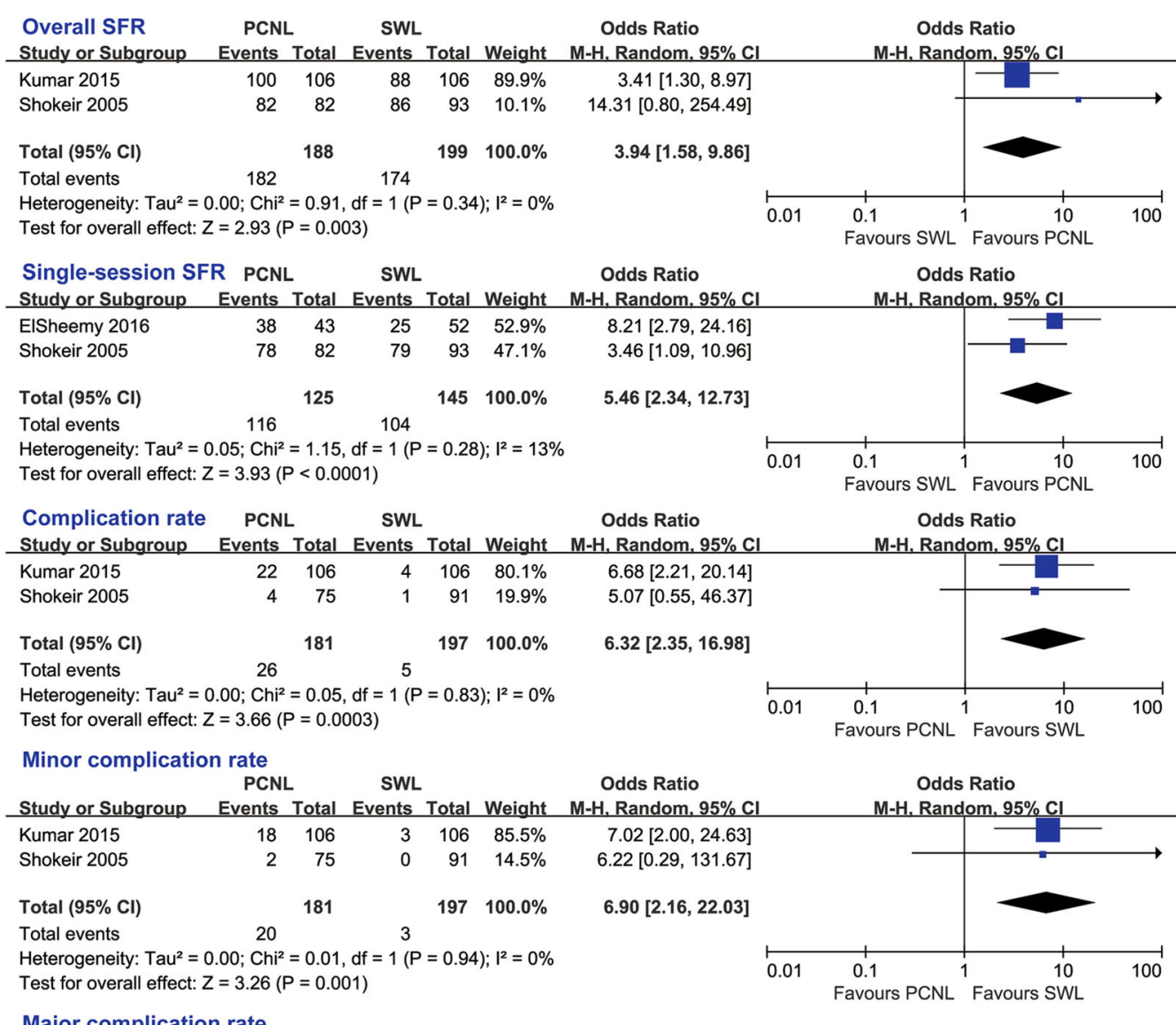

Major complication rate

$$
\text { PCNL }
$$

SWL

Odds Ratio

Odds Ratio

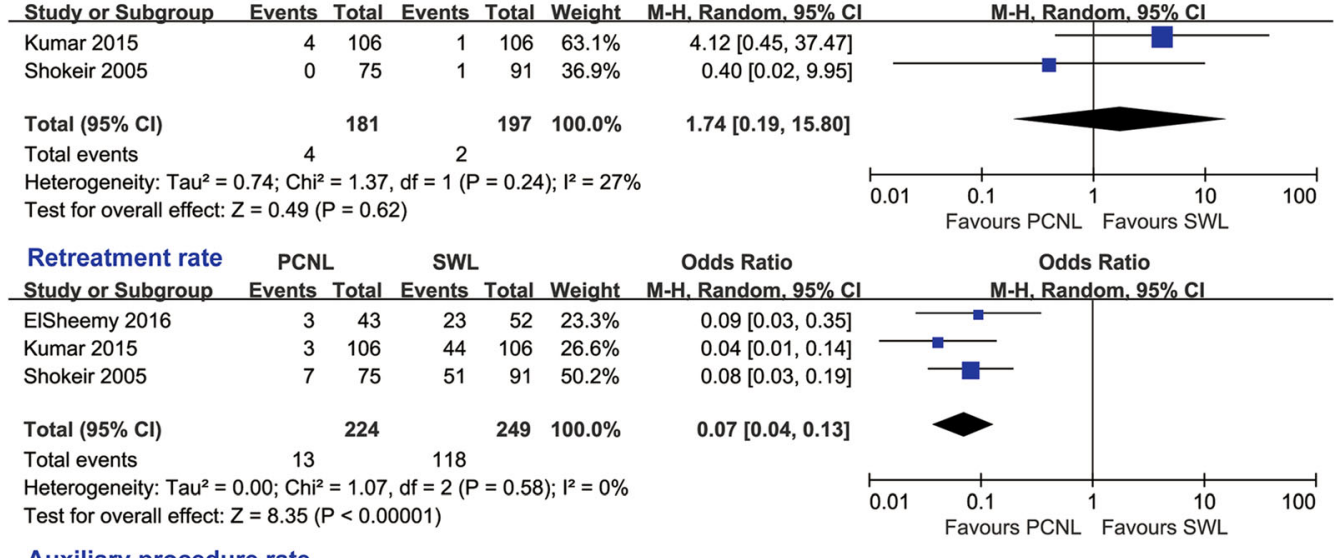

Auxiliary procedure rate

$$
\text { PCNL }
$$

SWL

Odds Ratio

Odds Ratio

Study or Subgroup Events Total Events Total Weight M-H. Random, 95\% C

$\begin{array}{llllll}\text { Kumar } 2015 & 6 & 106 & 15 & 106 & 61.1 \%\end{array}$

Shokeir 2005

$\begin{array}{rr}6 & 106 \\ 4 & 82\end{array}$

$8 \quad 93 \quad 38.9 \%$

$0.36[0.14,0.98]$

$0.54[0.16,1.88]$

Total $(95 \% \mathrm{Cl})$

188

$199 \quad 100.0 \%$

Total events

10 23

Heterogeneity: $\mathrm{Tau}^{2}=0.00 ; \mathrm{Chi}^{2}=0.25, \mathrm{df}=1(\mathrm{P}=0.62) ; \mathrm{I}^{2}=0 \%$ Test for overall effect: $Z=2.17(P=0.03)$

$0.43[0.20,0.92]$

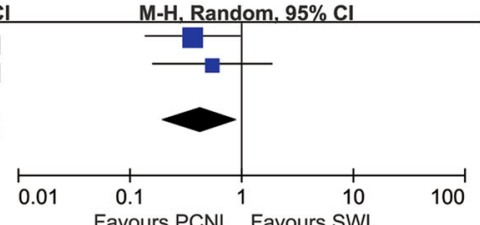

Fig. 7 Subgroup analyses results of PCNL vs SWL 


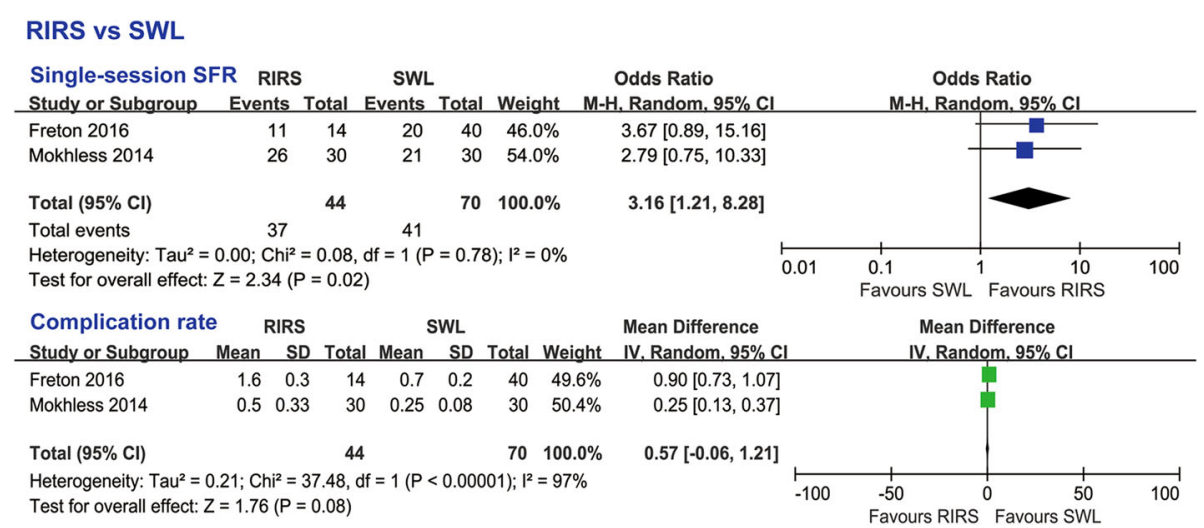

Fig. 8 Subgroup analyses results of RIRS vS SWL

PCNL (PCNL vs RIRS: $3.1 \pm 1.2$ vs $1.7 \pm 0.6$, days) [23]. PCNL requires the creation and dilation of an access tract through the renal parenchyma, and this is deemed to be more invasive than RIRS or SWL. However, the application of micro-PCNL or even super-mini PCNL can reduce complication events with a very high single session SFR.

SWL is considered the most minimally invasive procedure among these 3 treatment modalities, and it is still the first-line therapy for pediatric renal stones. In our analysis, RIRS is a more effective procedure than SWL according to the higher single-session SFR (OR 2.35, 95\% CI 1.21 to $4.55, p=0.05$ ) and higher overall SFR in pediatric patients with upper urinary tract stone size $\leq 20 \mathrm{~mm}$, although comparative studies of SWL vs RIRS included in this review had no positive result in SFR, including from an RCT [28]. Furthermore, the biological effects of SWL may induce acute injury of the renal parenchyma and adjacent tissues due to the acute effects of SWL, such as focal hemorrhage, rupture of small veins, extravasation and pooling of blood, necrosis in vasculature, disintegration in podocytes and mesangial cells, blood within Bowman's space and renal tubules, ischaemic changes, and infiltration by inflammatory cells [29]. Shock wave-induced transient tubular functional damage has been observed by Villanyi KK and colleagues [30]. Therefore, these authors recommended that consecutive treatments for pediatric renal stones should be spaced by at least 2 weeks. Although there is no clinical evidence about the long-term effect of SWL on pediatric kidneys [29], this effect should be kept in mind when SWL is chosen for pediatric renal stones due to the kidney still being in the growth and development stage.

In this review, higher overall complication rates and higher minor complication rates were only significantly found in the subgroup analyses of PCNL vs SWL. Although there was no significant difference in the various complication rates among the other subgroup analyses, blood transfusion was indicated exclusively in PCNL patients exclusively in our review. The highest transfusion rate, $13.64 \%$, was reported by Saad KS, et al. [24]. Desoky EA and his colleagues [31] claimed that PCNL in the pediatric age group via the flankfree modified supine position was safe and effective in the management of renal pelvis stones of sizes $20-30 \mathrm{~cm}$, and the SFR was similar to conventional PCNL in the prone position with only 1 (4.5\%) blood transfusion.

Except for the results of subgroup analyses, there were no significant differences in all of the indexes of the complication rates comparing SWL with the other two treatments. This finding disagrees with the widespread recognition that SWL is the most minimally invasive procedure among these techniques. Moreover, steinstrasse formation was only observed in SWL (1.1-25\%), except for 1 case of steinstrasse formation in PCNL [18] and another case in RIRS [22]. However, the fragment clearance after pediatric SWL is more efficient than in adults. D'Addessi A, et al. [28] indicated that, since the child's ureter is shorter than an adult's, this shorter length partially compensates for its narrower lumen, and the pediatric ureter is more elastic and distensible, promoting the passage of stone fragments. Finally, the small body volume of children reduces the loss of energy of the shockwaves in the process of release.

SWL had an undoubtedly shorter operative time than PCNL and RIRS, and it is usually performed as an outpatient treatment. All of the included studies preferred SWL over PCNL or RIRS, considering the operative time and hospital stay. However, with instrument miniaturization and other optimizations, RIRS has been increasingly performed, like SWL, as an outpatient procedure. The hospital stay of RIRS was also significantly shorter than PCNL, and this could reduce the medical cost, in spite of no significant difference being found in subgroup analyses. In addition to the relatively longer operative time and longer 


\section{PCNL vS RIRS}

\begin{tabular}{|c|c|c|c|c|c|}
\hline $\begin{array}{l}\text { Overall SFR } \\
\text { Study or Subgroup }\end{array}$ & $\begin{array}{c}\text { PCNL } \\
\text { Events }\end{array}$ & Total & $\begin{array}{l}\text { RIRS } \\
\text { Events }\end{array}$ & & Weight \\
\hline Bas 2016 & 36 & 45 & 31 & 36 & $61.8 \%$ \\
\hline Sen 2017 & 21 & 25 & 19 & 23 & $38.2 \%$ \\
\hline Total $(95 \% \mathrm{Cl})$ & & 70 & & 59 & $100.0 \%$ \\
\hline \multicolumn{6}{|c|}{$\begin{array}{l}\text { Heterogeneity: } \mathrm{Tau}^{2}=0.00 ; \mathrm{Chi}^{2}=0.30, \mathrm{df}=1(\mathrm{P}=0.58) ; \mathrm{I}^{2}=0 \% \\
\text { Test for overall effect: } \mathrm{Z}=0.49(P=0.63)\end{array}$} \\
\hline $\begin{array}{l}\text { Single-session SFR } \\
\text { Study or Subgroup }\end{array}$ & $\begin{array}{l}\text { PCNL } \\
\text { Events }\end{array}$ & Total & $\begin{array}{l}\text { RIRS } \\
\text { Events }\end{array}$ & & Weight \\
\hline Resorlu 2012 & 13 & 13 & 76 & 87 & $21.6 \%$ \\
\hline Sen 2017 & 21 & 25 & 19 & 23 & $78.4 \%$ \\
\hline Total $(95 \% \mathrm{Cl})$ & & 38 & & 110 & $100.0 \%$ \\
\hline Total events & 34 & & 95 & & \\
\hline \multicolumn{6}{|c|}{$\begin{array}{l}\text { Heterogeneity: } \mathrm{Tau}^{2}=0.00 ; \mathrm{Chi}^{2}=0.65, \mathrm{df}=1(\mathrm{P}=0.42) ; \mathrm{I}^{2}=0 \% \\
\text { Test for overall effect: } \mathrm{Z}=0.56(P=0.58)\end{array}$} \\
\hline
\end{tabular}

Odds Ratio

Odds Ratio

$\begin{array}{lrrrrr}\text { Study or Subgroup } & \text { Events Total } & \text { Events } & \text { Total Weight } & \text { M-H, Random, 95\% Cl }\end{array}$

M-H, Random, $95 \% \mathrm{Cl}$

$1.11[0.24,5.05]$

$0.79[0.31,2.03]$

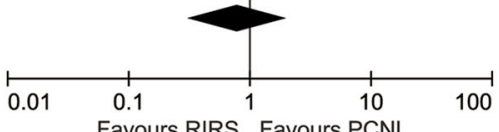

Odds Ratio

Favours RIRS Favours PCNL

-H. Random, $95 \% \mathrm{Cl}$

Odds Ratio

$4.06[0.23,73.03]$

$1.11[0.24,5.05]$

$1.46[0.38,5.62]$

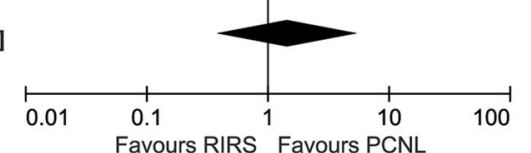

Complication rate PCNL RIRS

Odds Ratio

Study or Subgroup Events Total Events Total Weight M-H. Random, $95 \% \mathrm{Cl}$

$\begin{array}{lllllll}\text { Bas } 2016 & 6 & 45 & 6 & 36 & 63.5 \% & 0.77[0.23,2.63]\end{array}$

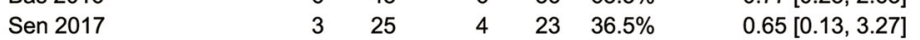

$\begin{array}{llll}\text { Total }(95 \% \mathrm{Cl}) & 70 & 59 & 100.0 \%\end{array}$

Total events $\quad 9 \quad 10$

Heterogeneity: $\mathrm{Tau}^{2}=0.00 ; \mathrm{Chi}^{2}=0.03, \mathrm{df}=1(\mathrm{P}=0.87) ; \mathrm{I}^{2}=0 \%$

Test for overall effect: $Z=0.65(P=0.51)$

$0.72[0.27,1.92]$

Minor complication rate

PCNL

RIRS

Odds Ratio

Odds Ratio

Study or Subgroup Events Total Events Total Weight M-H, Random, 95\% CI

$\begin{array}{lllllll}\text { Bas } 2016 & 5 & 45 & 5 & 36 & 59.8 \% & 0.78[0.21,2.92]\end{array}$

$\begin{array}{llllll}\text { Sen } 2017 & 3 & 25 & 4 & 23 & 40.2 \%\end{array}$

$0.65[0.13,3.27]$

Total $(95 \% \mathrm{Cl})$

70

$59100.0 \%$

Total events

8

9

$0.72[0.26,2.01]$

Heterogeneity: $\mathrm{Tau}^{2}=0.00 ; \mathrm{Chi}^{2}=0.03, \mathrm{df}=1(\mathrm{P}=0.87) ; \mathrm{I}^{2}=0 \%$

Test for overall effect: $Z=0.62(P=0.53)$

Operative time

PCNL

RIRS

M-H, Random, $95 \% \mathrm{Cl}$

Study or Subgroup Mean SD Total Mean SD Total Weight IV, Random, 95\%

\begin{tabular}{lllllllll} 
Bas 2016 & 57.11 & 15.75 & 45 & 53.25 & 15.08 & 36 & $57.9 \%$ & 3.86 \\
\hline & $-2.88,10.60]$
\end{tabular}

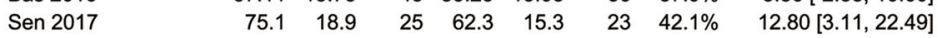

Total $(95 \% \mathrm{Cl})$

70

$59 \quad 100.0 \% \quad 7.62[-1.03,16.27]$

Heterogeneity: $\mathrm{Tau}^{2}=21.81 ; \mathrm{Chi}^{2}=2.20, \mathrm{df}=1(\mathrm{P}=0.14) ; \mathrm{I}^{2}=55 \%$

Test for overall effect: $Z=1.73(P=0.08)$

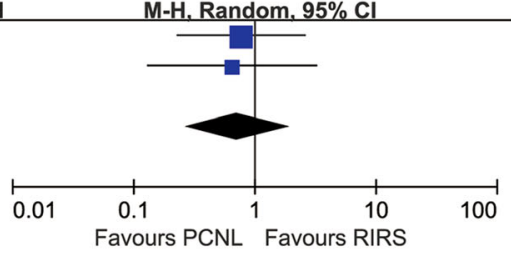

Fluoroscopy time PCNL RIRS Mean Difference

Study or Subgroup Mean SD Total Mean SD Total Weight IV, Random. $95 \% \mathrm{Cl}$

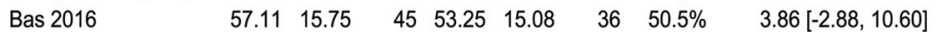

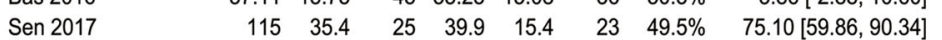

Total $(95 \% \mathrm{Cl}) \quad 70 \quad 59 \quad 100.0 \% 39.14\left[\begin{array}{ll}-30.67,108.95] & \end{array}\right.$

Heterogeneity: $\mathrm{Tau}^{2}=2501.44 ; \mathrm{Chi}^{2}=70.23, \mathrm{df}=1(\mathrm{P}<0.00001) ;\left.\right|^{2}=99 \%$

Test for overall effect: $Z=1.10(P=0.27)$

\begin{tabular}{|c|c|c|c|c|c|c|c|c|c|c|c|}
\hline \multirow{2}{*}{$\begin{array}{l}\text { Hospital stay } \\
\text { Study or Subgroup }\end{array}$} & \multicolumn{2}{|c|}{ PCNL } & \multicolumn{4}{|c|}{ RIRS } & \multirow{2}{*}{\multicolumn{2}{|c|}{$\begin{array}{ll} & \text { Mean Difference } \\
\text { Weight } & \text { IV. Random, } 95 \% \mathrm{Cl} \\
\end{array}$}} & \multicolumn{3}{|c|}{ (9) } \\
\hline & Mean & SD & Total & Mean & SD & Total & & & & IV. Random. $95 \% \mathrm{Cl}$ & \\
\hline Bas 2016 & 2.29 & 0.92 & 45 & 1.55 & 0.77 & 36 & $49.0 \%$ & $0.74[0.37,1.11]$ & & a & \\
\hline Total $(95 \% \mathrm{Cl})$ & & & 70 & & & 59 & $100.0 \%$ & $0.31[-0.51,1.13]$ & & & \\
\hline $\begin{array}{l}\text { Heterogeneity: } \mathrm{Tau}^{2}= \\
\text { Test for overall effect: }\end{array}$ & $\begin{array}{l}0.32 ; \mathrm{Cr} \\
\mathrm{Z}=0.74\end{array}$ & $\begin{array}{l}h i^{2}=12 \\
4(P=0\end{array}$ & $\begin{array}{l}2.46, \mathrm{df} \\
0.46)\end{array}$ & $=1(\mathrm{P}=$ & $=0.00$ & $04) ;\left.\right|^{2}=$ & $=92 \%$ & & -10 & $-50 \quad 0$ & 100 \\
\hline
\end{tabular}

Fig. 9 Subgroup analyses results of PCNL vs RIRS 
hospital stay, PCNL also presented a significantly longer fluoroscopy time than SWL or RIRS. Ristau BT and his colleagues [32] reported that radiation exposure was vital during treatment of pediatric stone disease, especially for defining the location of the stone when PCNL was performed. Therefore, these authors recommended that urologists should closely monitor the amount of radiation dose and limit the maximum effective dose to as low as reasonably achievable (ALARA) [33]. Specifically, ALARA principles mandated that the maximum effective dose should not exceed $50 \mathrm{mSv}$ in any 1-year period, and an average dose less than $20 \mathrm{mSv}$ per year over any 5 -year period. In brief, the urologists should devote themselves to reducing the radiation hazard as much as possible.

Since SWL is mainly performed under ultrasonographic guidance, it becomes a more attractive option with lower radiation exposure than the other two treatments. However, SWL exhibited a significantly higher retreatment rate compared with the other two therapies, while the retreatment rate between PCNL and RIRS did not show any significant difference. Furthermore, SWL also caused a significantly higher auxiliary procedure rate than PCNL. Considering that the fragment clearance of children is more rapidly than that of adults after SWL, surgical approaches seem to be a last resort. Soygur $\mathrm{T}$ and his colleagues [34] suggested that expectant management was usually efficient even in patients who developed steinstrasse after SWL, except for patients with larger stones $(>20 \mathrm{~mm})$, staghorn calculi or sepsis with an obstructed kidney. However, RIRS has a lower efficiency than SWL according to the EQ. In addition, every single EQ of RIRS from the included studies was less than $50 \%$ because the complete RIRS process includes one session for passive dilatation, one session for the lithotripsy and stent insertion, and one session for stent removal. Therefore, this technique requires more sessions under anaesthesia and more surgical risks.

When interpreting the results of our review, some limitations should be addressed. First, only 3 RCTs were available for final analysis, and more than half of the included studies were nonrandomized case control comparisons. Inevitably, patients and investigators cannot be double-blinded to the interventions. Allocation concealment was also not described in some RCTs. Furthermore, not all of the included articles were high-quality studies. A lack of more high-quality studies reduces the persuasiveness of our work. Second, no included study compared all three treatments head-to-head, and it was impossible to conduct a high-quality network meta-analysis since there were not enough relevant RCTs. Third, due to the nature of non-randomized studies, selection bias could be a major confounder, which might bias our conclusions.
Finally, the heterogeneity among the studies was obvious for several parameters, which might be a result of the differences in pediatric inclusive criteria, physical environment, surgical skills, outcome definitions and standards, or imaging during follow-up. Even more concerning is the stone burdens were not similar among these included studies, and there was not enough data to execute a sufficient subgroup analysis.

In spite of these limitations, our study still has some merits and values. To the best of our knowledge, this is the first systematic review that has simultaneously analysed the efficacy and safety of SWL, PCNL, and RIRS simultaneously in the treatment of pediatric upper urinary tract stones. Furthermore, our work, updated with the most recent data, provides a newly reference for the selection of the ideal treatment modality in pediatric urolithiasis. However, it is noteworthy that making an optimal recommendation is unusually difficult, since the clinical option is based on the stone size, stone location, patient age, instrument caliber, previous interventions history, comorbidity, and other factors.

\section{Conclusion}

In summary, our review suggests that SWL performed as an outpatient procedure provides a shorter hospital stay and operative time, with a lower SFR, higher retreatment rate, higher auxiliary procedure rate, and relative lower EQ. PCNL presents a higher SFR than SWL and the highest $\mathrm{EQ}$, but this is a technique accompanied by a longer fluoroscopy time and operative time than the other two modalities. RIRS offers a higher single-session SFR and lower retreatment rate than SWL but a lower EQ than SWL and PCNL, although a significantly shorter hospital stay than PCNL. Higher complication rates and higher minor complication rates were only significantly found in subgroup analyses of PCNL vs SWL. In other words, the complication rates are comparable among the three modalities, and most of these were minor complications.

The ultimate target of pediatric urinary stone management is to achieve extreme stone clearance with a safe and effective therapeutic regimen. To achieve this ideal target, urologists must choose the optimal individual modality, or combined other therapeutic regimens, according to the pediatric conditions and the goals of the parents.

\section{Abbreviations \\ ALARA: As low as reasonably achievable; AUA: American Urological Association; Cl: Confidence interval; EAU: Uropean Association of Urology; EQ: Effectiveness quotients; GFR: Glomerular filtration rate; LE: Level of evidence; NOS: Newcastle-Ottawa Scale; OR: Odds ratio; PCNL: Percutaneous nephrolithotomy; RCT: Randomized control trail; RIRS: Retrograde intrarenal surgery; SD: Standard deviation; SFR: Stone free rate; SWL: Extracorporeal shockwave lithotripsy; WMD: Weighted mean difference}

\section{Acknowledgements}

We thank Dr. Hasan Serkan Dogan and Dr. Ephrem Odoy Olweny for providing very useful comments during the revision of this study. 


\section{Authors' contributions}

$\mathrm{QH}$ and $\mathrm{KWX}$ carried out the literature search, quality assessment and data extraction, participated in the data analysis, drafted and revised the manuscript. YTC performed the statistical analysis and made the figures. BHL participated in the study design and supervised the project. HL and KJW conceived of the study and helped draft the manuscript. KJW is also the corresponding author for this manuscript. All authors read and approved the final manuscript

\section{Funding}

This study was supported by Grant No. 81470927 and No. 81770703 from the National Natural Science Foundation of China.

\section{Availability of data and materials}

All data analysed during this study are included in this published article.

\section{Ethics approval and consent to participate}

\section{Not applicable.}

\section{Consent for publication}

The results presented in this paper have not been published previously in whole or part, except in abstract form (36th World Congress of Endourology, Journal of Endourology. Sep 2018. https://doi.org/10.1089/end.2018.29043. abstracts). This publication has obtained permission from the copyright holder.

\section{Competing interests}

The authors declare that they have no competing interests.

Received: 15 May 2018 Accepted: 11 September 2019

Published online: 23 October 2019

\section{References}

1. Mahmud M, Zaidi Z. Percutaneous nephrolithotomy in children before school age: experience of a Pakistani Centre. BJU Int. 2004:94:1352-4.

2. Straub M, Gschwend J, Zorn C. Pediatric urolithiasis: the current surgical management. Pediatr Nephrol. 2010;25:1239-44.

3. Turk C, Petrik A, Sarica K, Seitz C, Skolarikos A, Straub M, et al. EAU guidelines on interventional treatment for urolithiasis. Eur Urol. 2016;69:475-82.

4. Assimos D, Krambeck A, Miller NL, Monga M, Murad MH, Nelson CP, et al. Surgical Management of Stones: American urological association/ Endourological society guideline, PART I. J Urol. 2016;196:1153-60.

5. Guven S, Istanbulluoglu O, Ozturk A, Ozturk B, Piskin M, Cicek T, et al. Percutaneous nephrolithotomy is highly efficient and safe in infants and children under 3 years of age. Urol Int. 2010;85:455-60.

6. Dogan HS, Kilicarslan H, Kordan Y, Celen S, Oktay B. Percutaneous nephrolithotomy in children: does age matter? World J Urol. 2011;29:725-9.

7. Dogan HS, Tekgul S. Minimally invasive surgical approaches to kidney stones in children. Curr Urol Rep. 2012;13:298-306.

8. Vanlangendonck R, Landman J. Ureteral access strategies: pro-access sheath Urol Clin North Am. 2004;31:71-81.

9. Safwat AS, Bissada NK, Kumar U, Taha MI, Abdel-Hafez SES, Eltaher AM, et al. Experience with ureteroscopic holmium laser lithotripsy in children. Pediatr Surg Int. 2008;24:579-81.

10. Clavien PA, Barkun J, de Oliveira ML, Vauthey JN, Dindo D, Schulick RD, et al. The Clavien-Dindo classification of surgical complications: five-year experience. Ann Surg. 2009;250:187-96.

11. Clayman RV, McClennan BL, Garvin TJ, Denstedt JD, Andriole GL. Lithostar: an electromagnetic acoustic shock wave unit for extracorporeal lithotripsy. J Endourol. 1989;3:307-13.

12. B. Phillips, C. Ball, D. Sackett, D. Banenoch, S. Straus, B. Haynes MD. Oxford Centre for Evidencebased Medicine-Levels of Evidence 2009. [Internet]. Cent. Evidence-Based Med. [cited 2015 Jan 5]. Available from: http://www. cebm.net/index.aspx? o $=1025$

13. Oremus M, Wolfson C, Perrault A, Demers L, Momoli F, Moride Y. Interrater reliability of the modified Jadad quality scale for systematic reviews of Alzheimer's disease drug trials. Dement Geriatr Cogn Disord. 2001;12:232-6.

14. Wells GA, Shea B, O'Connell D, Peterson J, Welch V, Losos M, et al. The Newcastle-Ottawa scale (NOS) for assessing the quality of nonrandomized studies in meta-analyses. Ottawa Hosp Res Inst. 2013. Available: http://www. ohri.ca/programs/clinical_epidemiology/oxford.asp.
15. Zeng G, Jia J, Zhao Z, Wu W, Zhao Z, Zhong W. Treatment of renal stones in infants: comparing extracorporeal shock wave lithotripsy and mini-percutaneous nephrolithotomy. Urol Res. 2012;40:599-603.

16. Wadhwa P, Aron M, Bal CS, Dhanpatty B, Gupta NP. Critical prospective appraisal of renal morphology and function in children undergoing shockwave lithotripsy and percutaneous nephrolithotomy. J Endourol. 2007;21:961-6.

17. Kumar A, Kumar N, Vasudeva P, Kumar R, Jha SK, Singh H. A single center experience comparing Miniperc and shockwave lithotripsy for treatment of radiopaque $1-2 \mathrm{~cm}$ lower Caliceal renal calculi in children: a prospective randomized study. J Endourol. 2015;29:805-9.

18. Hatipoglu NK, Sancaktutar AA, Tepeler A, Bodakci MN, Penbegul N, Atar M, et al. Comparison of shockwave lithotripsy and microperc for treatment of kidney stones in children. J Endourol. 2013;27:1141-6.

19. Shokeir AA, Sheir KZ, El-Nahas AR, El-Assmy AM, Eassa W, El-Kappany HA. Treatment of renal stones in children: a comparison between percutaneous nephrolithotomy and shock wave lithotripsy. J Urol. 2006;176:706-10.

20. ElSheemy MS, Daw K, Habib E, Aboulela W, Fathy H, Shouman AM, et al. Lower calyceal and renal pelvic stones in preschool children: a comparative study of mini-percutaneous nephrolithotomy versus extracorporeal shockwave lithotripsy. Int J Urol. 2016;23:564-70.

21. Mokhless IA, Abdeldaeim HM, Saad A, Zahran AR. Retrograde intrarenal surgery monotherapy versus shock wave lithotripsy for stones 10 to $20 \mathrm{~mm}$ in preschool children: a prospective, randomized study. J Urol. 2014;191:1496-9.

22. Freton L, Peyronnet B, Arnaud A, Tondut L, Hascoet J, Pradere B, et al. Extracorporeal shockwave lithotripsy versus flexible Ureteroscopy for the Management of Upper Tract Urinary Stones in children. J Endourol. 2017;31:1-6.

23. Resorlu B, Unsal A, Tepeler A, Atis G, Tokatli Z, Oztuna D, et al. Comparison of retrograde intrarenal surgery and mini-percutaneous nephrolithotomy in children with moderate-size kidney stones: results of multi-institutional analysis. Urology. 2012;80:519-23.

24. Saad KSM, Youssif ME, Al Islam Nafis Hamdy S, Fahmy A, El Din Hanno AG, El-Nahas AR. Percutaneous Nephrolithotomy vs retrograde intrarenal surgery for large renal stones in pediatric patients: a randomized controlled trial. J Urol. 2015;194:1716-20.

25. Bas O, Dede O, Aydogmus Y, Utangac M, Yikilmaz TN, Damar E, et al. Comparison of retrograde intrarenal surgery and micro-percutaneous Nephrolithotomy in moderately sized pediatric kidney stones. J Endourol. 2016;30:765-70.

26. Sen H, Seckiner I, Bayrak O, Dogan K, Erturhan S. A comparison of micro$P E R C$ and retrograde intrarenal surgery results in pediatric patients with renal stones. J Pediatr Urol. 2017;13:619.e1-5.

27. Pelit ES, Atis G, Kati B, Akin Y, Ciftci H, Culpan M, et al. Comparison of minipercutaneous Nephrolithotomy and retrograde intrarenal surgery in preschool-aged children. Urology. 2017;101:21-5.

28. D’Addessi A, Bongiovanni L, Sasso F, Gulino G, Falabella R, Bassi $P$. Extracorporeal shockwave lithotripsy in pediatrics. J Endourol. 2008;22:1-12.

29. Akin Y, Yucel S. Long-term effects of pediatric extracorporeal shockwave lithotripsy on renal function. Res Rep Urol. 2014;6(1):21-5.

30. Villanyi KK, Szekely JG, Farkas LM, Javor E, Pusztai C. Short-term changes in renal function after extracorporeal shock wave lithotripsy in children. J Urol. 2001;166:222-4.

31. Desoky EAE, EISayed ER, Eliwa A, Sleem M, Shabana W, Dawood T, et al. Flank-free modified supine percutaneous Nephrolithotomy in pediatric age group. Urology. 2015;85:1162-5.

32. Ristau BT, Dudley AG, Casella DP, Dwyer ME, Fox JA, Cannon GM, et al. Tracking of radiation exposure in pediatric stone patients: the time is now. J Pediatr Urol. 2015:11:339.e1-5.

33. Wrixon AD. New ICRP recommendations. J Radiol Prot. 2008;28(2):161-8.

34. Soygur T, Arikan N, Kilic O, Suer E. Extracorporeal shock wave lithotripsy in children: evaluation of the results considering the need for auxiliary procedures. J Pediatr Urol. 2006:2:459-63.

\section{Publisher's Note}

Springer Nature remains neutral with regard to jurisdictional claims in published maps and institutional affiliations. 